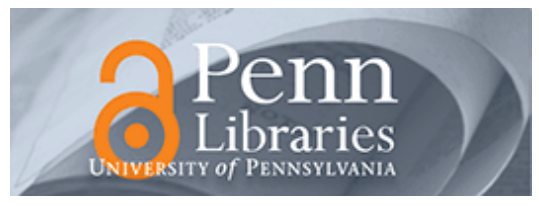

University of Pennsylvania

ScholarlyCommons

Marketing Papers

Wharton Faculty Research

$10-2010$

\title{
Competitive Consequences of Using a Category Captain
}

Upender Subramanian

Jagmohan S. Raju

University of Pennsylvania

Sanjay K. Dhar

Yusong Wang

Follow this and additional works at: https://repository.upenn.edu/marketing_papers

Part of the Business Administration, Management, and Operations Commons, Business and Corporate Communications Commons, Business Intelligence Commons, Marketing Commons, and the Organizational Behavior and Theory Commons

\section{Recommended Citation}

Subramanian, U., Raju, J. S., Dhar, S. K., \& Wang, Y. (2010). Competitive Consequences of Using a Category Captain. Management Science, 56 (10), 1739-1765. http://dx.doi.org/10.1287/mnsc.1100.1211

This paper is posted at ScholarlyCommons. https://repository.upenn.edu/marketing_papers/298

For more information, please contact repository@pobox.upenn.edu. 


\title{
Competitive Consequences of Using a Category Captain
}

\begin{abstract}
Many retailers designate one national brand manufacturer in each product category as a "category captain" to help manage the entire category. A category captain may perform demand-enhancing services such as better shelf arrangements, shelf-space management, and design and management of in-store displays. In this paper, we examine when and why a retailer may engage one manufacturer exclusively as a category captain to provide such service and the implications. We find that demand substitutability of competing brands gives rise to a service efficiency effect-service that expands the category is more effective in increasing a manufacturer's sales and margin than service that shifts demand from a rival's brand. We show that the service efficiency effect may motivate a category captain to provide a service that benefits all brands in the category even though doing so is more costly. We further show that, in categories that are less price competitive, there is higher competition between manufacturers to become the category captain. Consequently, a retailer may obtain better service by using a category captain than by engaging both manufacturers simultaneously. Our findings may help explain why a retailer may rely on a category captain despite concerns regarding opportunism and why there is limited empirical evidence of harm to rival manufacturers.
\end{abstract}

\section{Keywords}

category management, delegation, distribution channels, retailing, supply chain, collaboration

\section{Disciplines}

Business | Business Administration, Management, and Operations | Business and Corporate Communications | Business Intelligence | Marketing | Organizational Behavior and Theory 


\title{
The Competitive Consequences of Using a Category Captain
}

\author{
Sanjay K. Dhar
}

Jagmohan S. Raju

Upender Subramanian

Yusong Wang ${ }^{1}$

This version: May 2009

\footnotetext{
${ }^{1}$ Sanjay K. Dhar is James H. Lorie Professor of Marketing, Graduate School of Business, University of Chicago, Chicago, IL 60637 (Phone: (773) 702-3005, Fax: (773) 702-0458, Email: sanjay.dhar@chicagogsb.edu). Jagmohan S. Raju is Joseph J. Aresty Professor of Marketing, The Wharton School, University of Pennsylvania, Philadelphia, PA 19104 (Phone: (215) 898-1114, Fax: (215) 898-2534, E-mail: rajuj@wharton.upenn.edu). Upender Subramanian is a doctoral candidate in Marketing, The Wharton School, University of Pennsylvania, Philadelphia, PA 19104 (Phone: (215) 898-5252, Fax: (215) 898-2534, E-mail: upender@wharton.upenn.edu). Yusong Wang is Assistant Professor of Marketing, School of Management, Fudan University, P.R. China. (Email: yusongw@fudan.edu.cn). Correspondence about the paper should be addresed to upender@wharton.upenn.edu.
} 


\title{
The Competitive Consequences of Using a Category Captain
}

\begin{abstract}
Many retailers designate a national brand manufacturer as a "category captain" to help manage the entire category. The category captain is involved in decisions that affect not only its own brands, but also those of its rivals. We examine how engaging a self-interested category captain affects the category captain, the retailer and the rival manufacturer, when the category captain is responsible for providing retail service that can enhance demand (e.g., shelf-space design and allocation or managing end-of-aisle displays). We further examine when and why a retailer may rely on only one manufacturer as a category captain, even when more than one manufacturer can provide such service. We also examine characteristics that might make one manufacturer a more likely candidate for category captaincy than its rival.

We analyze a setting where two manufacturers supply to a single retailer, and the retailer may engage one or both manufacturers to provide retail service. Even in the absence of competition for category captaincy, we find that the category captain may still provide a service that enhances demand for all brands in the category. Moreover, even when the category captain's service depletes demand for the rival's brand, the rival manufacturer and the retailer may still benefit. When there is competition for category captaincy, we find that not only does the category captain provide a higher level of service, but also the service is less biased towards its own brand. Therefore, we find that the retailer may prefer the category captain arrangement over engaging both manufacturers jointly to provide service. Our findings may help explain why, despite concerns about the lack of category captain objectivity, this practice of relying on only one manufacturer has flourished, and why there is limited evidence of harm to rival manufacturers.
\end{abstract}

(Keywords: Retailing, Distribution Channels, Category Management.) 


\section{Introduction}

Category management can be defined as "a process that involves managing product categories as business units, and customizing them on a store-by-store basis to satisfy customer needs" (NMR and AMA 1992). Since its origins in the early 90's, category management has become an integral part of retail strategy both in the U.S. and in Europe (e.g., ACNielsen 2005, ECR 2006). As retailers carry hundreds or even thousands of product categories, they often do not find it practical to maintain dedicated resources or build specialized capabilities in each category. Instead, they rely on national brand manufacturers, as they usually have better insights about overall market trends, demand drivers and consumer shopping behavior. Retailers frequently partner with one leading national brand manufacturer in each category to help them manage the entire category (Blattberg and Fox 1995, FTC Report 2001). This is true not only for smaller retailers but also for large and leading ones such as Wal-Mart, Target and Safeway. For instance, Wal-Mart partnered with General Mills for the soy-milk category (Progressive Grocer 2006); Target with Cadbury Schweppes for premium beverages (Cadbury Schweppes 2005); and, Safeway with Campbell for soups (Campbell 2008). The partnering manufacturer is commonly referred to as the "category captain". Exhibit 1 provides some more examples that we obtained from managers at Safeway through an email survey.

\begin{tabular}{l|l|l|}
\cline { 2 - 3 } \multicolumn{1}{c|}{ Product Category } & Category Captain \\
\cline { 2 - 3 } 1) & Shelf Stable Juice \& Drinks & Ocean Spray \\
\cline { 2 - 3 } 2$)$ & Carbonated Soft Drinks & PepsiCo \\
\cline { 2 - 3 } 3$)$ & Pet Food \& Pet Needs & Nestle Purina \\
\cline { 2 - 3 } 4$)$ & Skin Care & Unilever \\
\cline { 2 - 3 } 5) & Paper - Bath Tissue, Paper & Georgia Pacific \\
\hline
\end{tabular}

\begin{tabular}{r|l|l|}
\cline { 2 - 3 } \multicolumn{1}{c|}{ Product Category } & Category Captain \\
\cline { 2 - 3 } 6) & General Purpose Cleaners & Clorox \\
\cline { 2 - 3 } 7$)$ & Canned Coffee / Hot & Kraft \\
\cline { 2 - 3 } 8$)$ & Food Prep/ Protection & Glad (Clorox) \\
\cline { 2 - 3 } 9$)$ & Diapers & P\&G \\
\cline { 2 - 3 } 10$)$ & Laundry Detergent & P\&G \\
\cline { 2 - 3 } & &
\end{tabular}

Exhibit 1: Category Captain Examples from Safeway (Source: Authors' E-mail Survey)

The category captain manufacturer is responsible for reviewing the performance of the entire category, providing information about category and brand level demand, and recommending a store-level sales strategy, including assortment, shelf-space assignments, promotion and pricing. For example, Kraft Foods demonstrated to retailers that changing the relative placement of pourable dressings and spoonable dressings can increase pourable category volume by $12.3 \%$ and spoonable 
dressing volume by 7.4\% (Supermarket News 2003). Similarly, Carrefour, a French global retail chain, realized a 6-16\% sales increase in the oral care category across retail markets based on Colgate's recommendations on relative product placements and educating consumers in-store (ECR Conference 2004). Interestingly, the scope of such arrangements extends beyond just the category captain's own brands, and also includes those of its rivals.

Retailers often ask manufacturers to compete for this position, and replace existing category captains with new ones if they are not satisfied with the outcomes. While a category captain devotes considerable resources to perform its role, it typically does not receive any direct compensation from the retailer (Carameli 2004). Rather, manufacturers are annually benchmarked based on their performance as category captains (e.g., Cannondale Associates 2008), and top performers are recognized through industry awards (e.g., Progressive Grocer 2008). Exhibit 2 shows the results of one such study. Such accolades can be a matter of pride and prestige within the industry.

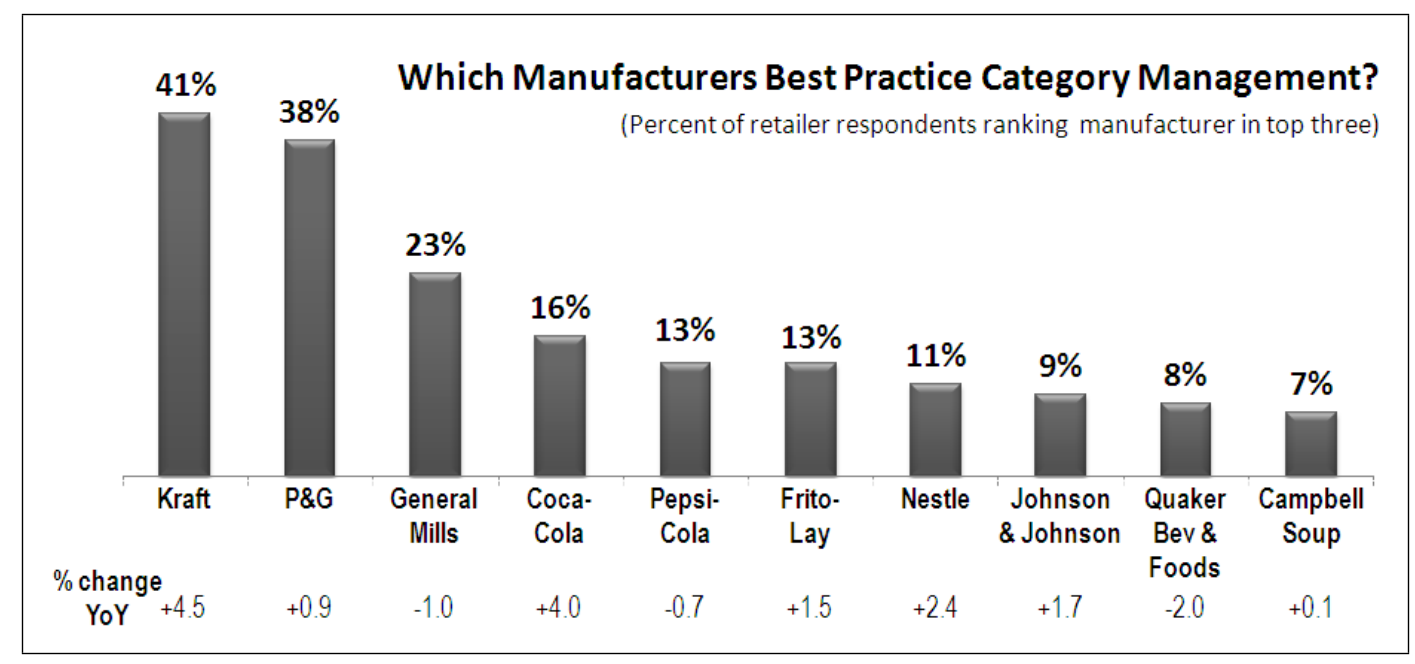

Exhibit 2: Manufacturer Benchmarking Survey (Cannondale Associates 2005)

More importantly, category captainship provides the manufacturer with some degree of decisionmaking authority over the retailer's strategy. While the level of influence may vary across categories and retailers, the category captain occupies a unique position relative to its rivals (Desrochers, Gundlach and Foer 2003, Lindblom and Olkkonen 2006). Indeed, according to an AC Nielsen survey (Progressive Grocer 2003), the most important reason for manufacturers to be involved in category management is to influence retailer category decisions. Consequently, category captainship is considered crucial for success and is a top-management priority for many leading national brand 
manufacturers. For instance, Clorox reports its category captain engagements and new category captain wins in its presentations to investors (see Exhibit 3). P\&G's COO identified increasing the number and scope of P\&G's category captain engagements as one of his top five challenges for 2007 (TRMM Workshop 2007).

\begin{tabular}{|c|c|c|c|c|c|c|c|c|c|c|} 
Category & $\begin{array}{c}\text { Home } \\
\text { Care }\end{array}$ & $\begin{array}{c}\text { Food Bags, } \\
\text { Wraps, Cntrs }\end{array}$ & $\begin{array}{c}\text { Trash } \\
\text { Bags }\end{array}$ & $\begin{array}{c}\text { Water } \\
\text { Filtration }\end{array}$ & Cat Litter & $\begin{array}{c}\text { Laundry } \\
\text { Additives }\end{array}$ & Charcoal & $\begin{array}{c}\text { Salad } \\
\text { Dressing }\end{array}$ & $\begin{array}{c}\text { BBQ \& } \\
\text { Marinades }\end{array}$ & $\begin{array}{c}\text { Auto } \\
\text { Care }\end{array}$ \\
\hline Retailer & Clorox & Glad & Glad & $\begin{array}{c}\text { Brita } \\
\text { Brita }\end{array}$ & $\begin{array}{c}\text { Fresh Step, } \\
\text { Scoop Away, } \\
\text { EverClean }\end{array}$ & Clorox & Kingsford & $\begin{array}{c}\text { Hidden } \\
\text { Valley }\end{array}$ & $\begin{array}{c}\text { KC } \\
\text { Masterpiece }\end{array}$ & $\begin{array}{c}\text { Armor } \\
\text { All, } \\
\text { STP }\end{array}$ \\
\hline Wal-mart & & & $\mathrm{X}$ & $\mathrm{X}$ & $\mathrm{X}$ & $\mathrm{X}$ & $\mathrm{X}$ & $\mathrm{X}$ & $\mathrm{X}$ \\
Target & & $\mathrm{X}$ & $\mathrm{X}$ & $\mathrm{X}$ & $\mathrm{X}$ & $\mathrm{X}$ & & & $\mathrm{X}$ \\
$\begin{array}{c}\text { Dollar } \\
\text { General }\end{array}$ & $\mathrm{X}$ & $\mathrm{X}$ & $\mathrm{X}$ & & $\mathrm{X}$ & $\mathrm{X}$ & $\mathrm{X}$ & & $\mathrm{X}$ \\
$\begin{array}{c}\text { Family } \\
\text { Dollar }\end{array}$ & $\mathrm{X}$ & $\mathrm{X}$ & $\mathrm{X}$ & & $\mathrm{X}$ & $\mathrm{X}$ & & & $\mathrm{X}$ \\
Safeway & $\mathrm{X}$ & $\mathrm{X}$ & $\mathrm{X}$ & & & $\mathrm{X}$ & $\mathrm{X}$ & & & $\mathrm{X}$ \\
SuperValu & $\mathrm{X}$ & & $\mathrm{X}$ & & $\mathrm{X}$ & $\mathrm{X}$ & $\mathrm{X}$ & & & \\
\hline
\end{tabular}

$\mathrm{X}$ : Clorox designated as category captain.

Exhibit 3: Clorox's Category Captain Assignments by Retailer and Category (Clorox 2009)

However, if the intent of category captains is to influence retailers, and the scope of their role involves managing the entire category including their rival's brands, then this raises some obvious concerns. While the use of category captains per se is not considered illegal or anticompetitive by regulatory authorities (e.g., FTC Report 2001), researchers in marketing (e.g., Gruen and Shah 2000, Morgan, Kaleka and Gooner 2007) as well as legal scholars (e.g., Wright 2006, Carameli 2004) have expressed doubts about the objectivity of the category captain and worry about the potential harm to retailer and rival manufacturers' interests ${ }^{2}$. As Carameli observes,

"Intuitively, the category captain relationship is like the fox guarding the henhouse...

After all, the manufacturer is concerned primarily with its own brands and has a vested interest in seeing competing products fail... there is a fundamental difference between the interest of the manufacturer and those of the retailer it serves."

Some retailers share these concerns and are wary of category captains being biased towards their own brands (e.g., Supermarket News 2007, FTC Roundtable 2003). Understandably, this underlying

\footnotetext{
${ }^{2}$ For instance, in the U.S., there is no federal regulation on category captaincy. The FTC only recommends guidelines to minimize the potential for anticompetitive conduct such as the retailer making the final category management decisions, limiting the competitive information that goes to the category captain and requiring category captains to establish managerial firewalls within their organizations (FTC Report 2001).
} 
difference of interests has led researchers and industry analysts to question the rationale for using category captains and doubt its prospects (e.g., Gruen and Shah 2000, McKinsey 1997).

Yet, this practice has flourished. It has emerged as the dominant mode of category management for many retailers in the U.S. and in Europe, and has started making inroads in emerging markets, such as Brazil and India (e.g., Arkader and Ferreira 2004, Business Line 2006). Furthermore, despite concerns about the category captains using their position to disadvantage their rivals, there is limited evidence of such bias or harm to rival manufacturers (Leary 2003). For instance, in their survey of U.K. retailers, Morgan, Kaleka and Gooner (2007) find that the average level of category captain opportunism reported is relatively low, as is the extent of discontent amongst non-category captain manufacturers. Similarly, in their surveys of Finnish suppliers, Lindblom and Olkkonen (2006) find that the non-category captain manufacturers tend to be on an average neutral rather than negative about the impact of category captain arrangements on their market performance.

Our objective in this paper is to conduct a model-based examination to understand how category management, when performed by a self-interested category captain, affects the category captain, the retailer and the rival manufacturer. Also of key interest is to explain why a retailer may find it desirable to engage only one of the manufacturers to provide category management services that enhance demand. These services could include shelf design, shelf-space allocation, design of end-of-aisle displays, stocking of products that customers desire, and inventory management such that demand does not go unfulfilled. These are amongst the most common category management initiatives undertaken by manufacturers and retailers (ACNielsen 2005), and are also the areas where category captains have held the most decision making responsibility (Lindblom and Olkkonen 2006). In addition to such demand enhancing activities, category captains may also be responsible for other activities such as providing superior demand information to the retailer and making retail pricing decisions. While we do not examine all possible activities a category captain may be asked to perform, we believe our research makes a useful start and that our results are potentially useful to retailers, manufacturers and policy makers.

We analyze a market with two national brand manufacturers and one retailer. The retailer may engage one or both manufacturers to provide demand enhancing retail service. A manufacturer may provide a service that boosts its own demand at the expense of its rival's. Or, it may provide a service that also boosts the rival's demand, though it may be more costly to do so. We develop 
our analysis as follows:

1. In $\S 3$, we assume that only one particular manufacturer is able to provide service, and hence does not face competition for category captaincy. We study the nature and impact of the category captain's service when there is ample scope for the category captain to be biased.

2. In $\S 4$, we consider that both manufacturers can offer service, but the service cannot be offered by both simultaneously (e.g., shelf-space management). We examine how competition for category captaincy impacts the service and when the category captain arrangement is more beneficial.

3. In $\S 5$, we examine conditions under which a retailer may engage only one manufacturer even when it is feasible to engage both manufacturers simultaneously. Thus, we study why the use of a category captain arrangement may emerge as an equilibrium outcome.

4. In $\S 6$, to identify characteristics that might make one manufacturer a more likely candidate for category captaincy, we consider that the manufacturers are asymmetric.

Table 1 provides an overview of our analysis and the questions addressed at each stage.

Key Results: When only one particular manufacturer can provide service, we find that the retailer engages that manufacturer as a category captain even though the manufacturer may deploy its service so as to boost only its demand at the expense of its rival's. Interestingly, even when the category captain's service depletes the rival manufacturer's demand, we find that the rival manufacturer may still benefit as long as the service is not too biased. More importantly, even in the absence of competition for category captaincy, we find that the category captain may still provide a service that enhances demand for all brands in the category.

When there is competition for category captaincy, we find that the category captain's service is less biased, thereby increasing the scope for the rival manufacturer to benefit. However, it is also possible that, in their bid to become the category captain, the manufacturers compete away their profits and are worse off than without the arrangement. Thus, in certain product categories, the category captain arrangement might simply become the cost of doing business with the retailer. Even when the retailer has the alternative of engaging both manufacturers jointly to provide service, we find that the retailer may still choose to engage only one of them, since competition for category captaincy can lead to better service provision than when the manufacturers are jointly engaged. 


\begin{tabular}{|c|c|c|}
\hline Section & Setting & Key Questions Addressed \\
\hline \multicolumn{3}{|c|}{ Without Competition for Category Captaincy } \\
\hline$\S 3$ & $\begin{array}{l}\text { Only one manufacturer can } \\
\text { offer service. }\end{array}$ & $\begin{array}{l}\text { How does engaging a self-interested category captain } \\
\text { affect the retailer and rival manufacturer? }\end{array}$ \\
\hline \multicolumn{3}{|c|}{ With Competition for Category Captaincy } \\
\hline$\S 4$ & $\begin{array}{l}\text { Both manufacturers can of- } \\
\text { fer service, but joint service } \\
\text { is not feasible. }\end{array}$ & $\begin{array}{l}\text { How does competition for category captaincy affect } \\
\text { the outcome? When is the category captain arrange- } \\
\text { ment more beneficial? }\end{array}$ \\
\hline \multicolumn{3}{|c|}{ Category Captaincy When Joint Provision of Service is Feasible } \\
\hline$\S 5$ & $\begin{array}{l}\text { Both manufacturers can of- } \\
\text { fer service, and joint service } \\
\text { is feasible. }\end{array}$ & $\begin{array}{l}\text { When should a retailer engage only one of the manu- } \\
\text { facturers to provide retail service? }\end{array}$ \\
\hline \multicolumn{3}{|c|}{ Choice of the Category Captain } \\
\hline$\S 6$ & $\begin{array}{l}\text { Manufacturers are asymmet- } \\
\text { ric and can both offer service, } \\
\text { but not jointly. }\end{array}$ & $\begin{array}{l}\text { What characteristics make one manufacturer a more } \\
\text { likely candidate for category captaincy than its rival? }\end{array}$ \\
\hline
\end{tabular}

Table 1: Overview of Analysis

Related Research on Category Management: Much of the prior research on the impact of category management has been primarily concerned with the transition from a brand focus to a category focus; Zenor (1994) examines the impact on individual manufacturers with multi-brand product lines, Basuroy, Mantrala and Walters (2001) analyze the impact on retailers' prices and performance and Gajanan, Basuroy and Beldona (2007) investigate the impact on manufacturers' brand differentiation decisions. The use of category captains for the delegation of pricing decisions has been studied by Wang, Raju and Dhar (2003) and Kurtulus and Toktay(2007) under different conditions. Our work complements their research by examining category captaincy for non-price related demand enhancing activities, when there is competition for category captaincy and when manufacturers may also be jointly engaged for category management. Researchers have also examined the role and conduct of category captains using managerial surveys. In addition to Morgan, Kaleka and Gooner (2007) and Lindblom and Olkkonen (2006), Gruen and Shah (2000) investigate the determinants and outcomes of plan objectivity and implementation through a survey of 
manufacturer category managers in the U.S.

In what follows, we describe our model in $\S 2$. In $\S 3$ and $\S 4$, respectively, we analyze settings without and with competition for category captaincy. In $\S 5$, we examine when the retailer may still rely on the category captain arrangement even though joint provision of service is feasible. We examine model extensions in $\S 6$ and conclude in $\S 7$.

\section{Model Description}

We consider a mature product category with two national brands, $N B_{1}$ and $N B_{2}$, sold by manufacturers $M_{1}$ and $M_{2}$, respectively, in a retail outlet owned by retailer $R . M_{1}$ and $M_{2}$ supply their brands to the retailer at wholesale prices $w_{1}$ and $w_{2}$, respectively. We assume that the retailer's objective is to maximize the combined profits from both brands. We focus on one product category and abstract away from category substitution and complementarity effects. We do not model retailer competition.

The retailer may engage one or both manufacturers to provide retailing service that can enhance demand, such as better shelf space management, designing end-of-aisle displays, stocking products that the customers desire, and superior inventory management so that demand does not go unfulfilled. We now describe the settings when the retailer engages (i) neither manufacturer, (ii) one of the manufacturers and, (iii) both manufacturers to provide such retail service, noting that the third alternative may not be always feasible ${ }^{3}$.

Retailer Engages Neither Manufacturer For Retail Service: In the absence of retail service, we assume the demand to be,

$$
\begin{aligned}
& q_{1}=1-\frac{\theta}{1-\theta}\left(p_{1}-p_{2}\right)-p_{1}, \\
& q_{2}=1-\frac{\theta}{1-\theta}\left(p_{2}-p_{1}\right)-p_{2},
\end{aligned}
$$

where $q_{1}$ and $q_{2}$ are the unit sales, and $p_{1}$ and $p_{2}$ are the retail prices of $N B_{1}$ and $N B_{2}$, respectively. $\theta \in(0,1)$ is the degree of cross-price sensitivity between the brands, such that $\frac{\theta}{1-\theta}$ is the cross-price sensitivity. This formulation for cross-price sensitivity allows us to graphically depict the results

\footnotetext{
${ }^{3}$ As we explain in more detail in the respective sections, we consider in $\S 3$ that only $M_{1}$ is able to offer service, and in $\S 4$ that joint service is not feasible.
} 
in a concise manner, and to span the entire parameter range when computing results numerically. The demand functions in (1) and (2) have their basis in utility theory (e.g., Shubik and Levitan 1980, Singh and Vives 1984). Later, in $\S 6.1$, we allow for asymmetry in the base level of demand.

Retailer Engages One Manufacturer For Retail Service: In this setting the retailer engages only one of the manufacturers to provide retail service, i.e., the retailer relies on a category captain. The Colgate and Kraft examples described in $\S 1$ suggest that some demand enhancing activities may benefit all brands in a category. For instance, the category captain might convince the retailer to expand shelf space for the entire category, or improve the presentation of the entire category such that it increases demand for all brands. On the other hand, a frequent concern raised by some researchers and industry observers (e.g., Gruen and Shah 2000, Carameli 2004) is that a category captain may deploy these services in a manner that benefits the category captain's brand potentially at the expense of the rival's brand. For example, the category captain may allocate lesser shelf space to the rival's brand, relegate it to less visible portions on the shelf, or install an end-of-aisle display for its own brand. We allow the model to be flexible enough to accommodate either of these possibilities and let the manufacturer acting as the category captain to decide the nature of its service.

We decompose the impact of retailing service into an own-brand effect and a rival-brand effect. Let $\left(e_{i}, \rho_{i}\right)$ denote the service provided by $M_{i}$ when it is engaged as the category captain, such that the demand is given by,

$$
\begin{aligned}
q_{i} & =\left[1-\frac{\theta}{1-\theta}\left(p_{i}-p_{j}\right)-p_{i}\right]+\frac{1}{2} e_{i} \\
q_{j} & =\left[1-\frac{\theta}{1-\theta}\left(p_{j}-p_{i}\right)-p_{j}\right]+\frac{1}{2} \rho_{i} e_{i}
\end{aligned}
$$

for $i, j \in\{1,2\}, i \neq j$, where $e_{i}>0$ is the service level, and $\rho_{i} \in[-1,1]$ is the service bias that represents the direction and magnitude of impact that $M_{i}$ 's service has on its rival's demand. Thus, while the own-brand effect, $\frac{1}{2} e_{i}$, is always positive, the nature and extent of the rival brand effect, $\frac{1}{2} e_{i} \rho_{i}$, depends on the service bias. A positive $\rho_{i}$ indicates that $M_{i}$ 's service also enhances its rival's demand. On the other hand, a negative $\rho_{i}$ indicates that $M_{i}$ 's service depletes its rival's demand in the process of enhancing its own demand. We refer to a service with a higher (lower) $\rho_{i}$ as being "less biased" ("more biased"). 
Providing such retailing service is costly. The category captain must set up a dedicated team of managers and analysts, conduct market research and market trials to design and test retailing strategies, and develop and roll-out retailer-specific category plans. We assume that manufacturers are equally efficient in providing service. Later, in $\S 6.2$, we allow their efficiencies to differ.

Let $C(e, \rho)$ be the cost of providing a service $(e, \rho)$. We consider that the cost of service is increasing in both the own-brand effect and the rival-brand effect. We assume the following parsimonious quadratic-additive formulation for $C(e, \rho)$ that exhibits this characteristic:

$$
C(e, \rho)=\frac{1}{2} e^{2}+\frac{1}{2}\left[\frac{k}{1-k}\right][e(1+\rho)]^{2},
$$

where the first term corresponds to the own-brand effect and the second term corresponds to the rival-brand effect (with $\rho$ shifted by 1 so that this cost component is strictly increasing with the rival-brand effect). Other functional forms are also possible, but left for future research. In $\S 7$, we discuss the extent to which our results may be robust to other formulations.

Since the cost of service is increasing in both the own-brand effect and the rival-brand effect, it is more costly to increase one's demand when this is not solely at the expense of the rival's demand, i.e., it is more costly when $\rho>-1$ than when $\rho=-1$. In (5), the extent to which this is so is determined by $k \in\left[\frac{1}{3}, 1\right)^{4}$. Thus, when the category captain deploys its service to increase its demand by $\frac{1}{2} e$ solely at the expense of its rival $(\rho=-1)$, then it costs $\frac{1}{2} e^{2}$. But when this increase in demand is achieved only partially at the expense of its rival or while simultaneously increasing the rival's demand $(\rho>-1)$, then it costs an additional $\frac{1}{2}\left[\frac{k}{1-k}\right](1+\rho)^{2} e^{2}$. This additional cost increases with $\rho$, and increases faster when $k$ is higher. In other words, $k$ deteremines the extent to which it is costlier to increase one's demand not solely at the expense of the rival's demand. Thus, we refer to $k$ as the degree of difficulty to provide a less-biased service. $k$ is likely to depend on both the category management "technology" available to the manufacturer as well as the nature of the product category. For instance, it may be higher in more mature product categories.

Retailer Engages Both Manufacturers For Retail Service: As an alternative to the category captain arrangement, a retailer may consider engaging both manufacturers simultaneously,

\footnotetext{
${ }^{4}$ This $\left[\frac{k}{1-k}\right]$ formulation allows us to graphically depict the results in a concise manner, and to span the entire parameter range when computing results numerically. We restrict $k \geq \frac{1}{3}$ to ensure that the cost is sufficiently convex in $\rho$.
} 
or jointly, to provide retail service. We note that this may not be feasible for all activities. For instance, two manufacturers cannot both make decisions regarding shelf space allocation and placement. This is the situation in $\S 4$, where service provision is feasible only under a category captain arrangement. On the other hand, a retailer could have both manufacturers manage in-store displays at different locations within the store. We model this in $\S 5$. Further, we recognize that even when joint assignment is feasible, this may come at the loss of economies of scope and / or scale. For instance, it is likely to be more difficult for a manufacturer to achieve the same demand enhancing impact when it manages only half the in-store displays. More generally, category captaincy may allow for a wider range of demand enhancing activities than does joint assignment. Therefore, we consider that a manufacturer may be less efficient in providing service when jointly engaged along with its rival than when assigned as the category captain.

When $M_{1}$ and $M_{2}$ provide service of $\left(e_{1}^{J}, \rho_{1}^{J}\right)$ and $\left(e_{2}^{J}, \rho_{2}^{J}\right)$, respectively, under joint assignment, we take the demand to be given by,

$$
\begin{aligned}
& q_{1}=\left[1-\frac{\theta}{1-\theta}\left(p_{1}-p_{2}\right)-p_{1}\right]+\frac{1}{2} e_{1}^{J}+\frac{1}{2} \rho_{2}^{J} e_{2}^{J}, \\
& q_{2}=\left[1-\frac{\theta}{1-\theta}\left(p_{2}-p_{1}\right)-p_{2}\right]+\frac{1}{2} e_{2}^{J}+\frac{1}{2} \rho_{1}^{J} e_{1}^{J} .
\end{aligned}
$$

Thus, we consider that each manufacturer's service impacts demand independent of its rival's service. For both manufacturers, we take $\mu \in(0,1]$ to be the relative efficiency of providing service jointly as compared to providing service as the category captain arrangement, such that the cost of providing a service $(e, \rho)$ under joint assignment is $\frac{1}{\mu} C(e, \rho)$. If $\mu=1$, service provision under joint assignment is as efficient as under category captaincy. As $\mu$ decreases, joint service provision becomes relatively less efficient. As $\mu \rightarrow 0$, we approach the situation where joint service is infeasible.

\section{Without Competition for Category Captaincy}

One of the most discussed aspects of the category captain arrangement is the potential for category captain opportunism - the tendency for a category captain to deploy its service in a manner that benefits its own brand at the expense of its rival's - and the resulting harm to the rival manufacturer 
and the retailer. To examine whether and when these concerns are relevant, we start with a setting that presents the maximum scope for such opportunism. We arbitrarily select one manufacturer, say $M_{1}$, and consider that only $M_{1}$ is able to provide service and hence does not face competition for category captaincy.

This setting may also represent the situation in markets that are in their initial stages of adoption of category management, where, for some product categories, only one manufacturer may possess the ability to act as a category captain. For instance, in emerging markets such as Brazil and India, retailers have only recently begun to embrace category management, and often at the behest of a leading manufacturer (e.g., Arkader and Ferreira 2004, Business Line 2006). We model the interactions in this setting as follows:

Stage 1 Proposal for category captaincy: $M_{1}$ proposes the service $\left(e_{1}, \rho_{1}\right)$ it would provide if selected as the category captain.

Stage 2 Category captaincy decision: Retailer either accepts or rejects $M_{1}$ 's proposal. We denote this decision by $r \in\{0,1\}$, with $r=1$ if $M_{1}$ 's proposal is accepted, and $r=0$ otherwise.

Stage 3 Service provision: If $M_{1}$ is chosen as the category captain, then it provides the service it proposed in Stage 1. Else, there is no service provision.

Stage 4 Wholesale price decisions: Manufacturers simultaneously set their wholesale price $w_{i}$. Stage 5 Retail price decision: Retailer sets retail prices $p_{i}$.

In Stage 1, we could instead consider that $M_{1}$ commits to the profits the retailer will realize if $M_{1}$ were the category captain. In the absence of competing proposals, we find that this yields the same outcome. In Stage 2 , when $M_{1}$ proposes to offer service $\left(e_{1}>0\right)$, if the retailer is indifferent between accepting and rejecting the proposal, we assume that it accepts the proposal ${ }^{5}$. While there are no strategic decisions in Stage 3, we include it as a separate stage to make the timing of actions explicit. In this stage, we assume that $M_{1}$ provides the service it committed to in Stage 1. As we will see in our analysis, this will not be a concern as the equilibrium proposal is self-enforcing. In Stage 4, manufacturers set their wholesale price, taking into consideration the demand enhancing measures, if any, when the retailer appoints a category captain. In Stage 5, the retailer sets the

\footnotetext{
${ }^{5}$ If $e_{1}=0$, then there is no change in market demand, and the situation is identical to not having a category captain. We assume that the retailer rejects the proposal in this case.
} 
retail prices given the category captain arrangement and the wholesale prices.

We assume that all demand and cost parameters are common knowledge. We also assume that the manufacturers and the retailer make their decisions in order to maximize their respective profits. The retailer's profits are given by

$$
\Pi_{R}=\left(p_{1}-w_{1}\right) q_{1}+\left(p_{2}-w_{2}\right) q_{2}
$$

$M_{1}$ 's and $M_{2}$ 's profits, respectively, are given by

$$
\begin{aligned}
& \Pi_{1}=w_{1} q_{1}-\delta(r=1) C\left(e_{1}, \rho_{1}\right), \\
& \Pi_{2}=w_{2} q_{2},
\end{aligned}
$$

where $\delta(x)$ is an indicator function which equals one if $x$ is true and zero otherwise.

We solve for the subgame perfect Nash equilibrium. We are interested in, (i) when category captaincy occurs, (ii) what service the category captain provides, and (iii) how the rival manufacturer and the retailer are affected. We start by solving for the equilibrium in the subgames starting at Stage 3, where the retailer rejects $M_{1}$ 's proposal $(r=0)$ and where it accepts the proposal $(r=1)$. Given any proposal that $M_{1}$ may have made in Stage 1 , let $\Pi_{R}^{k}$ denote the retailer's equilibrium profits in the subgame starting at Stage 3 where the retailer has chosen the option $r=k, k \in\{0,1\}$, $i \in\{1,2\}$. Similarly, let $\Pi_{i}^{k}$ denote $M_{i}$ 's equilibrium profits in the subgame where the retailer has chosen the option $r=k, k \in\{0,1\}, i \in\{1,2\}$. Let $\Pi_{R}^{k *}$ and $\Pi_{i}^{k *}$ denote $\Pi_{R}^{k}$ and $\Pi_{i}^{k}$, respectively, in an an equilibrium of the overall game, $k \in\{0,1\}, i \in\{1,2\}$. Let $\left(e_{1}^{*}, \rho_{1}^{*}\right)$ denote $M_{1}$ 's equilibrium proposal. Lemma C.1 in Appendix C (pg 48) provides the equilibrium profits in the subgames. From this, we have,

Lemma 1. In the setting without competition for category captaincy, the retailer accepts $M_{1}$ 's proposal if $e_{1}>0 . M_{2}$ benefits from $M_{1}$ 's category captaincy iff $\rho_{1}>-\frac{\theta}{2}$. Total category demand is higher under $M_{1}$ 's category captaincy if $\rho_{1}>-1$.

Proof. Refer Appendix A (pg 38).

We find that the retailer always benefits from $M_{1}$ 's service, even if $M_{1}$ 's service boosts $N B_{1}$ 's demand solely at the expense of $N B_{2}$ 's, i.e., $\rho_{1}=-1$. When $\rho_{1}=-1$, relative to the case without 
$M_{1}$ 's service, the retailer can increase its average retail price while keeping category sales volume at the same level. It can achieve this by simultaneously increasing the price for the higher volume $N B_{1}$ and decreasing the price of the lower volume $N B_{2}$. Therefore, $M_{1}$ 's service allows the retailer to increase its average retail price for the same category sales volume ${ }^{6}$.

But at the same time, $M_{1}$ 's service also enables $M_{1}$ to increase its wholesale price, resulting in a higher average wholesale price for the retailer. This is despite $M_{2}$ reducing its wholesale price, since $N B_{1}$ 's sales volume is higher than $N B_{2}$ 's. Therefore, by accepting $M_{1}$ 's proposal, the retailer also faces a higher average wholesale price. We find that the increase in average retail price more than offsets the increase in average wholesale price ${ }^{7}$. Hence, the retailer benefits from $M_{1}$ 's service even when $\rho_{1}=-1$. When $\rho_{1}>-1, M_{1}$ 's service not only leads to a higher average retail margin, but also to a higher category sales volume. Thus, the retailer again benefits from $M_{1}$ 's service.

More interestingly, we find that $M_{2}$ may benefit from the category captain arrangement even if $M_{1}$ 's service depletes its demand, i.e., even when $\rho_{1}<0$. When $M_{1}$ provides service, the retailer charges a higher retail price for $N B_{1}$ than for $N B_{2}$ in order to increase its margin on the higher volume brand. Moreover, $M_{1}$ charges a higher wholesale price than $M_{2}$ does, and the retailer passes through some of this wholesale price differential. Thus, $M_{1}$ 's service leads to a higher retail price for $N B_{1}$ relative to $N B_{2}$, which causes demand to switch to $N B_{2}$. Consequently, in addition to its direct negative effect, $M_{1}$ 's service has an indirect positive effect on $N B_{2}$ 's demand through its effect on retail prices. We find that as long as $M_{1}$ 's service is not too biased $\left(\rho_{1}>-\frac{\theta}{2}\right)$, the latter effect offsets the former. Furthermore, the scope for $M_{2}$ to benefit from $M_{1}$ 's category captaincy increases with cross-price sensitivity, since higher cross-price sensitivity results in more demand switching in equilibrium ${ }^{8}$.

$N B_{1}$ 's demand is always higher under $M_{1}$ 's category captaincy, whereas $N B_{2}$ 's demand is higher only if $\rho_{1}>-\frac{\theta}{2}$. While we cannot comment on how category captaincy affects consumer welfare, since we do not explicitly model how demand enhancing activities affect consumer preferences (especially when $\rho_{1}<0$ ), we find that the total category demand increases as long as $\rho_{1}>-1$.

Since the retailer accepts $M_{1}$ 's proposal whenever $e_{1}>0, M_{1}$ bases its proposal solely on its

\footnotetext{
${ }^{6}$ All else being equal, this is, in fact, the retailer's best response.

${ }^{7}$ When $\rho_{1}=-1$, the equilibrium category sales volume is the same as without $M_{1}$ 's service.

${ }^{8}$ The equilibrium retail price differential between $N B_{1}$ and $N B_{2}$ decreases with cross-price sensitivity. But the extent of demand switching, given by $\frac{\theta}{1-\theta}\left(p_{1}-p_{2}\right)$, increases.
} 
own profits. Thus, the proposal is self-enforcing in that $M_{1}$ does not have an incentive to deviate from it once its proposal is accepted. Lemma 2 provides $M_{1}$ 's equilibrium proposal.

Lemma 2. In the setting without competition for category captaincy, $M_{1}$ 's equilibrium proposal is given by

$$
e_{1}^{*}=\frac{2 k(2-\theta)(1-\theta)(2+\theta)}{4 k\left(15+2 \theta-9 \theta^{2}+\theta^{4}\right)-\theta^{2}(1-\theta)} \quad, \quad \rho_{1}^{*}=\frac{\theta-2 k}{k(2-\theta)}
$$

Proof. Refer Appendix A (pg 38).

Since $M_{1}$ places its own interest above that of the other parties, one might expect that it would deploy its service so as to favor its brand at the expense of its rival's. Interestingly, we find that this is not always the case. We find that $M_{1}$ benefits from providing a less-biased service since this leads to a higher wholesale and retail price for $N B_{2}$, which in turn allows $M_{1}$ to charge a higher wholesale price. In other words, a less-biased service reduces the extent of price competition with the rival brand. Therefore, it is in $M_{1}$ 's own interest to provide a less-biased service, provided it is not too costly to do so.

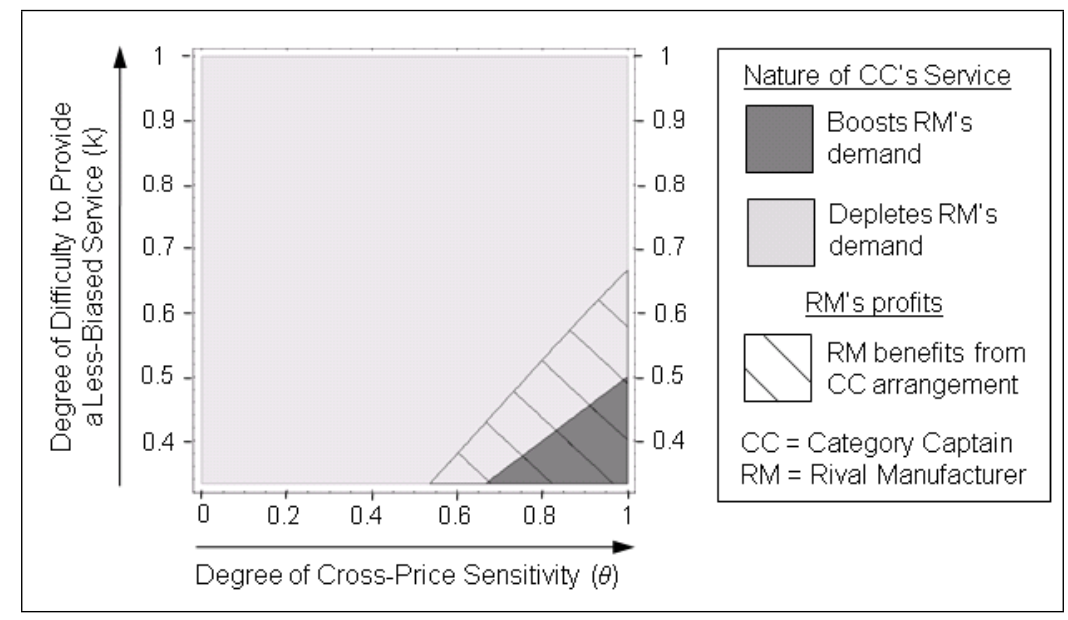

Figure 1: Outcomes In the Absence of Competition for Category Captaincy.

Furthermore, this effect is more pronounced when cross-price sensitivity is higher. Thus, when cross-price sensitivity is sufficiently high and the cost of providing a less-biased service is not too high $\left(\theta>\frac{2}{3}\right.$ and $\left.k<\frac{\theta}{2}\right)$, we find that, even in the absence of competition for category captaincy, the category captain provides a service that also boosts its rival's demand. Figure 1 depicts the outcomes as a function of $\theta$ and $k$, and the dark-shaded region corresponds to the instances where the category captain's service also boosts the rival manufacturer's demand. 
Clearly, in such instances, as $\rho_{1}>0$, the rival manufacturer stands to benefit from the category captain arrangement. In fact, as we already know from Lemma 1, the rival manufacturer benefits over a larger set of instances, i.e., whenever $\rho_{1}>-\frac{\theta}{2}$. These instances are shown as the hatched region in Figure 1. As cross-price sensitivity increases, not only does the scope for the rival manufacturer to benefit increase (Lemma 1), but also the service provided by the category captain is less biased (Lemma 2). Consequently, for both these reasons, the potential for the rival manufacturer to benefit increases with cross-price sensitivity. The rival manufacturer is also more likely to benefit when $k$ is low, since this allows for a less-biased service. Proposition 1 summarizes our findings.

Proposition 1. In the setting without competition for category captaincy,

(i) the retailer always engages a category captain and benefits from the arrangement.

(ii) the total category demand is higher than without the category captain arrangement.

(iii) the category captain always benefits from the arrangement. The category captain provides a service that also increases the rival's demand when cross-price sensitivity is sufficiently high and the cost of providing a less-biased service is sufficiently low $\left(\theta>\frac{2}{3}\right.$ and $k<k_{1}$, where $\left.k_{1}=\frac{\theta}{2}\right)$.

(iv) the rival manufacturer may benefit from the arrangement even if the category captain's service depletes its demand. Specifically, the rival manufacturer benefits when cross-price sensitivity is sufficiently high and the cost of providing a less-biased service is not too high $\left(\theta>\theta_{2}\right.$ and $k<k_{2}$, where $\theta_{2}=4-2 \sqrt{3}<\theta_{1}$ and $\left.k_{2}=\frac{2 \theta}{4-2 \theta+\theta^{2}}>k_{1}\right)$.

Proof. Refer Appendix B (pg 44).

Researchers and industry observers often raise concerns that lack of objectivity or opportunism on part of the category captain will harm the retailer and non-category captain manufacturers. However, researchers have also found limited evidence of such opportunism or harm to other manufacturers (Morgan, Kaleka and Gooner 2007, Lindblom and Olkkonen 2006). Our results above may explain why. Firstly, we find that it may be in the category captain's own interest to provide a service that also boosts the rival's demand. Secondly, even if the category captain's service depletes the rivals' demand, the rival manufacturer may still benefit from the category captain arrangement. Lastly, we find that the retailer stands to benefit from the arrangement even if the category captain 
is biased towards its own brand at the expense of the rival's. Further, while we cannot comment on how category captaincy affects consumer welfare, since we do not explicitly model how demand enhancing activities affect consumer preferences, total category demand always increases.

\section{With Competition for Category Captaincy}

In markets where category management is a well established practice, such as the U.S. and Europe, retailers often invite proposals for category captaincy from more than one manufacturer and select one. Having examined the scope for category captain opportunism, we now investigate how competition for category captaincy affects the nature of service provided. Such competition also raises the question whether the category captain ultimately benefits from the category captain arrangement. Further, we would like to know when the arrangement is more beneficial for the manufacturer and for the retailer. To investigate these issues, we consider the following setting where both manufacturers can provide service and make competing proposals for category captaincy:

Stage 1 Proposal for category captaincy: $M_{1}, M_{2}$ simultaneously propose the service $\left(e_{i}, \rho_{i}\right)$ they would provide if selected as the category captain.

Stage 2 Category captaincy decision: Retailer either accepts one of their proposals or rejects both. We denote this decision by $r \in\{0,1,2\}$, with $r=i$ if $M_{i}$ 's proposal is accepted, $i \in\{1,2\}$, and $r=0$ otherwise.

Stage 3 Service provision: If $M_{i}$ is chosen as the category captain, then it provides the service it proposed in Stage 1. If neither manufacturer was chosen as the category captain, then there is no service provision.

Stage 4 Wholesale price decisions: Manufacturers simultaneously set their wholesale price $w_{i}$. Stage 5 Retail price decision: Retailer sets retail prices $p_{i}$.

In Stage 1, we could instead consider that manufacturers commit to the profits the retailer would realize if they were made the category captain. We find that this leads to qualitatively similar insights, but is analytically more complex and defer this analysis to the Technical Appendix (pg 53). In Stage 2, we assume that if the retailer prefers to accept a proposal over rejecting both, but is indifferent between the two proposals, then it chooses either manufacturer with equal probability. In Stage 3, as before, we assume that a manufacturer is committed to the proposal 
it makes in Stage 1. In practice, if a manufacturer fails to keep its commitment, then it faces the prospects of not being reappointed as the category captain, and the position may instead be awarded to the rival manufacturer (Dupre \& Gruen 2004). We assume that this is a sufficient deterrent to ensure commitment. The retailer's profits are given by

$$
\Pi_{R}=\left(p_{1}-w_{1}\right) q_{1}+\left(p_{2}-w_{2}\right) q_{2} .
$$

$M_{1}$ 's and $M_{2}$ 's profits, respectively, are given by

$$
\begin{aligned}
& \Pi_{1}=w_{1} q_{1}-\delta(r=1) C\left(e_{1}, \rho_{1}\right), \\
& \Pi_{2}=w_{2} q_{2}-\delta(r=2) C\left(e_{2}, \rho_{2}\right),
\end{aligned}
$$

where $\delta(x)$ is an indicator function which equals one if $x$ is true and zero otherwise. We solve for the subgame perfect Nash equilibrium of the above game.

Given any pair of proposals that the manufacturers may have made in Stage 1 , let $\Pi_{R}^{k}$ and $\Pi_{i}^{k}$, respectively, denote the retailer's and $M_{i}$ 's equilibrium profits in the subgame starting at Stage 3 where the retailer has chosen the option $r=k, k \in\{0,1,2\}$. For $k \in\{1,2\}, \Pi_{R}^{k}$ is a function of $M_{k}$ 's proposal, but we denote this explicitly only where necessary. Let $\Pi_{R}^{k *}$ and $\Pi_{i}^{k *}$ denote $\Pi_{R}^{k}$ and $\Pi_{i}^{k}$, respectively, in an equilibrium of the overall game, $k \in\{0,1,2\}, i \in\{1,2\}$. Let $\left(e_{i}^{*}, \rho_{i}^{*}\right)$ denote $M_{i}$ 's equilibrium proposal.

Finally, let $\xi_{C C}, \xi_{R M}$ and $\xi_{R}$ denote the equilibrium profits of the category captain (CC), the rival manufacturer $(\mathrm{RM})$ and the retailer $(\mathrm{R})$, respectively, in the setting of $\S 3$, without competition for category captaincy.

\section{Conditions for Equilibrium}

The equilibrium wholesale and retail price strategies in the subgames starting in Stage 4 can be

solved in the usual manner. In Stage 2, the retailer chooses the option that offers it the most profits (with the tie-breaking rules mentioned earlier). Given the retailer's best response, let $\Omega_{i}$ denote 
$M_{i}$ 's profits in Stage $1, i \in\{1,2\}$. We then have,

$$
\Omega_{i}=\left\{\begin{array}{lll}
\Pi_{i}^{0}, & \text { if } & \Pi_{R}^{0}>\max \left\{\Pi_{R}^{1}, \Pi_{R}^{2}\right\} \\
\Pi_{i}^{1}, & \text { if } & \Pi_{R}^{1} \geq \Pi_{R}^{0}, \Pi_{R}^{1}>\Pi_{R}^{2} \\
\Pi_{i}^{2}, & \text { if } & \Pi_{R}^{2} \geq \Pi_{R}^{0}, \Pi_{R}^{2}>\Pi_{R}^{1} \\
\frac{1}{2}\left(\Pi_{i}^{1}+\Pi_{i}^{2}\right), & \text { if } & \Pi_{R}^{1}=\Pi_{R}^{2} \geq \Pi_{R}^{0} .
\end{array}\right.
$$

Let $\Omega_{i}^{*}$ denote $\Omega_{i}$ in an equilibrium of the overall game.

For $i, j \in\{1,2\}, i \neq j$, in an equilibrium where $M_{i}$ 's proposal is accepted with positive probability, we require that:

(i) the retailer accepts $M_{i}$ 's proposal:

$$
\Pi_{R}^{i *} \geq \max \left\{\Pi_{R}^{0 *}, \Pi_{R}^{j *}\right\}
$$

(ii) $M_{i}$ (weakly) prefers to win category captaincy over losing it:

$$
\begin{aligned}
& \text { if } \Pi_{R}^{j *}<\Pi_{R}^{0 *} \text {, then } \Omega_{i}^{*} \geq \Pi_{i}^{0 *} ; \\
& \text { if } \Pi_{R}^{j *} \geq \Pi_{R}^{0 *} \text {, then } \Omega_{i}^{*} \geq \Pi_{i}^{j *} \text {. }
\end{aligned}
$$

If $M_{i}$ were to give up its bid for category captaincy, its profits depend on the retailer's next best option. (16a) and (16b) ensure that, depending on the retailer's next best option, $M_{i}$ is (weakly) better off winning category captaincy.

(iii) $M_{i}$ 's proposal maximizes its profits, conditional on the retailer accepting the proposal:

$$
\begin{aligned}
\left(e_{i}^{*}, \rho_{i}^{*}\right) \in \underset{e_{i}, \rho_{i}}{\operatorname{argmax}} & \Omega_{i}, \\
\text { s.t. } & \Pi_{R}^{i} \geq \max \left\{\Pi_{R}^{0 *}, \Pi_{R}^{j *}\right\} .
\end{aligned}
$$

Along with the previous condition, this ensures that $M_{i}$ 's equilibrium proposal is indeed its best response. 
(iv) $M_{j}$ (weakly) prefers not to make a proposal that displaces $M_{i}$ from category captaincy:

$$
\begin{aligned}
\Omega_{j}^{*} \geq & \max _{e_{j}, \rho_{j}} \\
& \Omega_{j}, \\
& \text { s.t. } \quad \Pi_{R}^{j} \geq \max \left\{\Pi_{R}^{0 *}, \Pi_{R}^{i *}\right\} .
\end{aligned}
$$

The above conditions are not only necessary but also sufficient since they ensure that neither the retailer nor the manufacturers have an incentive to deviate.

\section{Active Competition for Category Captaincy}

We now define the notion of active competition that will be useful in characterizing the outcomes. In any equilibrium where $M_{i}$ 's proposal is accepted with positive probability, we say that there is active competition for category captaincy if

$$
\begin{gathered}
\Omega_{i}^{*}<\max _{e_{i}, \rho_{i}} \Pi_{i}^{i}=\xi_{C C}, \\
\text { s.t. } \Pi_{R}^{i} \geq \Pi_{R}^{0 *} .
\end{gathered}
$$

The right hand side of the inequality is the maximum profits that $M_{i}$ makes in the absence of a competing proposal from its rival, i.e., when only $M_{i}$ can offer service. This is given by $\xi_{C C}$. When (19) holds, it indicates that, in making its proposal, $M_{i}$ is constrained by its rival's proposal and consequently makes lower profits than in the absence of a competing proposal. In other words, when there is active competition in equilibrium, $M_{i}$ 's proposal is driven by the prospect of losing category captaincy to its rival. This can be seen by comparing the maximization problem in (17) with that in (19); the former has an additional constraint, which must be more binding for (19) to hold ${ }^{9}$. It follows that, with active competition the retailer's profits are higher than in the setting without competition for category captaincy. It also follows that in the absence of active competition, the equilibrium outcome is the same as when only $M_{i}$ can offer service.

Thus, we have a different outcome than in the setting without competition for category captaincy only when there is active competition. We now examine when this occurs.

\footnotetext{
${ }^{9}$ The rival's proposal must be sufficienty attractive for there to be active competition. It is possible that (19) does not hold even if $\Pi_{R}^{j *}>\Pi_{R}^{0 *}$, i.e., the retailer's next best option is to accept $M_{j}$ 's proposal and yet there is no active competition for category captaincy.
} 


\section{Equilibrium with Active Competition}

We solve for the equilibrium from the conditions in (15) - (18). From our analysis of the setting in $\S 3$ without competition for category captaincy, it follows that, if one manufacturer does not propose to offer service, then the other manufacturer always will and this proposal is always accepted by the retailer. Thus, category captaincy always occurs in equilibrium. Intuitively, one might expect that if it is more attractive for a manufacturer to become the category captain than let its rival become one, then the manufacturers would compete for category captaincy . We find that this is indeed the case.

Lemma 3. In the setting with competition for category captaincy, category captaincy always occurs in equilibrium. Further,

(i) if $\xi_{C C}>\xi_{R M}$, then there is a unique equilibrium, and there is active competition in this equilibrium;

(ii) if $\xi_{C C} \leq \xi_{R M}$, then there exist only equilibria without active competition, and, in any equilibrium where $M_{i}$ is chosen as the category captain with positive probability, $M_{i}$ 's equilibrium proposal is the same.

Proof. Refer Appendix A (pg 38).

Thus, we have a unique equilibrium whenever there is active competition, and degenerate equilibria in the absence of active competition. Degeneracy arises since, without active competition, the category captain's proposal is not driven by its rival's proposal. Hence slightly perturbing the rival's proposal does not affect the outcome. But the category captain's proposal in any such equilibrium is unique. Moreover, equilibria with and without active competition occur in mutually exclusive and exhaustive instances.

Suppose that, in the absence of competition for category captaincy, a manufacturer's profits as the category captain are more than as the non-category captain manufacturer, i.e., $\xi_{C C}>\xi_{R M}$. Then, either manufacturer would rather become the category captain than let its rival take over. As a result, in the setting when manufacturers can compete for category captaincy, we find that there is active competition in equilibrium.

On the other hand, suppose a manufacturer's profits as the non-category captain manufacturer 
are more than as the category captain, i.e., $\xi_{C C} \leq \xi_{R M}$. In other words, even without the threat of a competing proposal, the category captain's service is benevolent enough so that, after accounting for service costs, the non-category captain manufacturer is better off than the category captain. Then, either manufacturer would rather its rival become the category captain. Therefore, even when manufacturers can compete for category captaincy, we have equilibria without active competition. In fact, we find that this is the only type of equilibria when $\xi_{C C} \leq \xi_{R M}$.

While there is a multiplicity of equilibria in the case without active competition, we find that all equilibria where one particular manufacturer is chosen as the category captain lead to the same outcome. The degeneracy arises since slightly perturbing the rival's proposal does not affect the outcome. For instance, consider an equilibrium without active competition where (only) $M_{1}$ is chosen as the category captain. It follows that the constraint $\Pi_{R}^{i} \geq \Pi_{R}^{j *}$ in (17) is not binding (or only weakly binding). In other words, $M_{1}$ 's proposal is not driven by $M_{2}$ 's proposal. Then, we can construct another equilibrium with the same outcome, by slightly changing $M_{2}$ 's proposal such that equilibrium conditions (15) and (16b) still hold. The outcomes in all such equilibria are the same as when only $M_{1}$ can offer service.

Similarly, all equilibria where (only) $M_{2}$ is chosen as the category captain lead to the same equilibrium outcome as when only $M_{2}$ can offer service. Further, owing to the symmetry between the manufacturers, this equilibrium outcome can be obtained by starting with the equilibrium where $M_{1}$ is chosen as the category captain and switching the roles of the two manufacturers. Thus, in the case of equilibria without active competition, we can talk of a common equilibrium outcome if we ignore the identity of the manufacturer chosen as the category captain ${ }^{10}$. We adopt this approach for the rest of our discussion in this section.

Proposition 2. In the setting with competition for category captaincy, there is active competition for category captaincy if cross-price sensitivity is sufficiently low or if the cost of providing a lessbiased service is sufficiently high (if $\theta<\theta_{3}$ or, if $\theta \geq \theta_{3}$ and $k>k_{3}$, where $\theta_{3} \approx 0.73$ and $k_{3}$ is defined in Appendix B).

Proof. Refer Appendix B (pg 44).

\footnotetext{
${ }^{10}$ When $\xi_{C C}=\xi_{R M}$, there also exists an equilibrium where either manufacturer is chosen as the category captain with equal probability. But $M_{i}$ 's proposal in this equilibrium is the same as its proposal in the equilibria where only $M_{i}$ is chosen as the category captain. Consequently, the equilibrium profits are the same in either case. They only differ in the outcomes in terms of the identity of the category captain.
} 
Figure 2 shows the results graphically. The region within the dashed line indicates active competition. We had seen that in the absence of competition for category captaincy, the rival manufacturer benefits from the category captain's service if $\theta>\theta_{2}$ and $k<k_{2}$ (Proposition 1). In fact, if $\theta>\theta_{3}$ and $k<k_{3}$, for $\theta_{3}>\theta_{2}$ and $k_{3}<k_{2}$, then the rival manufacturer benefits more from the arrangement than it would if it were to take over category captaincy itself, i.e., $\xi_{C C} \leq \xi_{R M}$. Therefore, there is no active competition for category captaincy in such instances.

In all other instances, we have active competition. Since each manufacturer would rather be the category captain than let its rival take over, the manufacturers engage in a bidding contest, where they attempt to outbid each other by proposing a service that offers higher profits for the retailer than does their rival's service. This continues up to the point where it is equally attractive for either of them to win category captaincy as it is to lose it. In equilibrium, we find that both manufacturers propose the same service and become the category captain with equal probability.

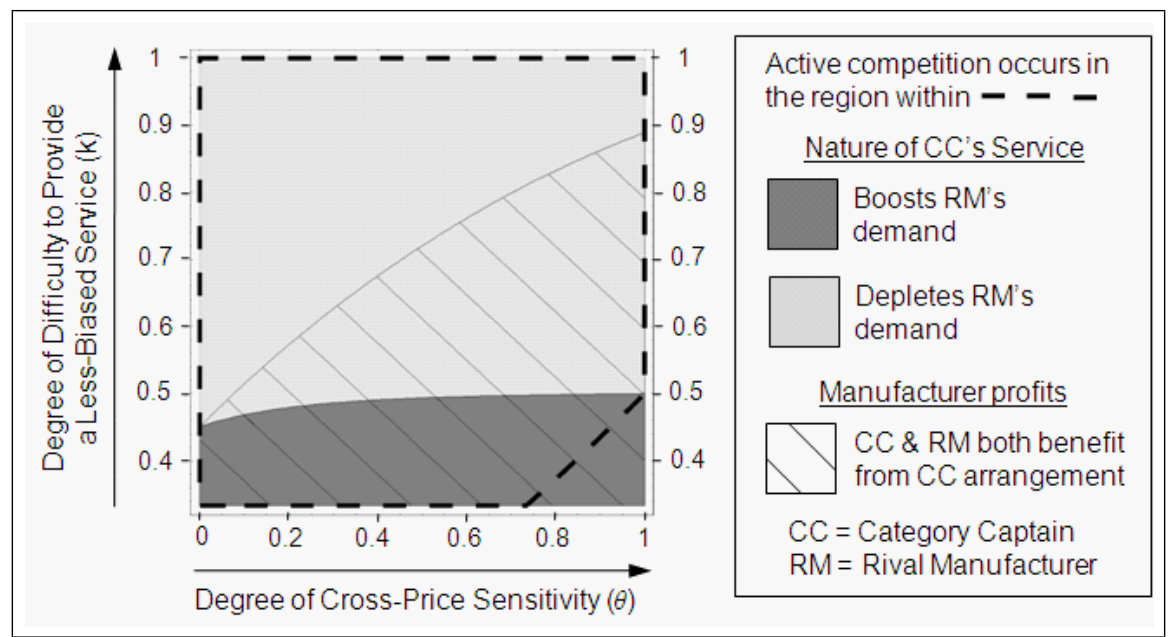

Figure 2: Outcomes in the Presence of Competition for Category Captaincy

\section{Impact of Active Competition on Retailing Service}

When there is active competition, a manufacturer must propose a service that provides as much profits to the retailer as does its rival's proposed service. In particular, it must provide more profits to the retailer than in the case without competing proposals. We find that this results in a higher level of service relative to the setting without competition for category captaincy. More interestingly, it also leads to a service that is less biased. 
Proposition 3. When there is active competition for category captaincy, the category captain's service is less biased and the service level is higher than in the setting without competition for category captaincy.

Proof. Refer Appendix B (pg 45).

While the category captain benefits only indirectly from a less-biased service through its effect on price competition, the retailer benefits more directly from its effect on the rival brand's demand. Moreover, the retailer does not incur the cost of providing a less-biased service. Consequently, when the manufacturer must provide higher profits to the retailer than in the setting without competition for category captaincy, we find that it is worthwhile for it to offer a service that is less biased.

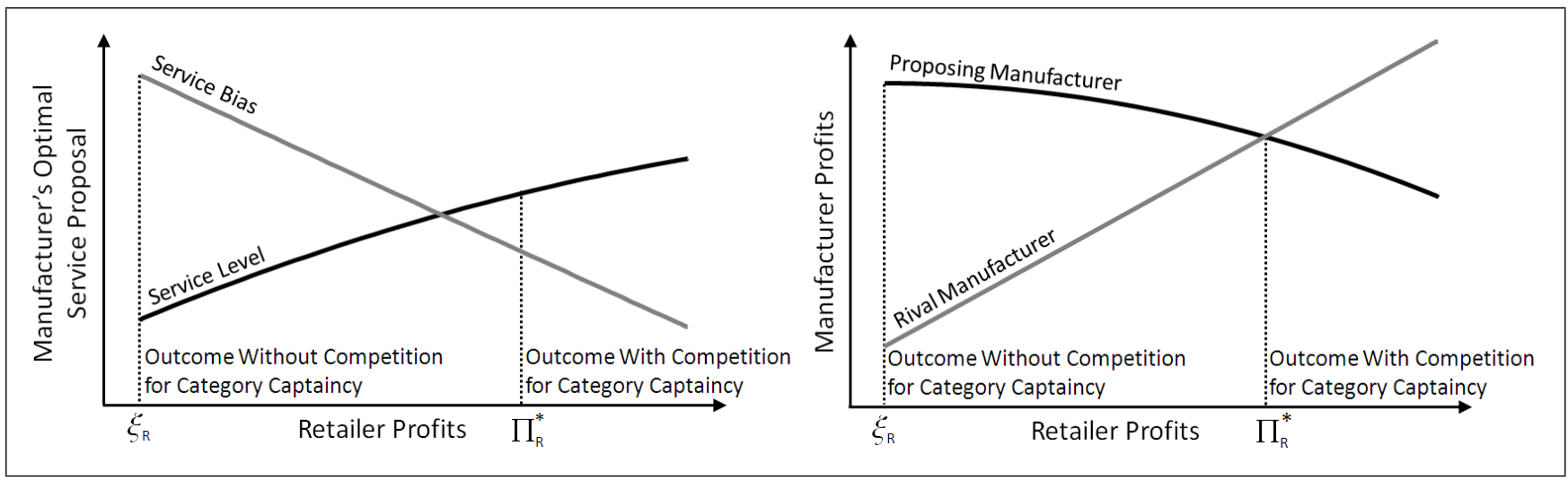

Figure 3: Optimal service proposal and corresponding manufacturer profits, for a given level of retailer

$$
\text { profits }^{11} \text {. }
$$

Figure 3 illustrates the nature of strategic interactions that we have seen thus far under active competition. The panel on the left depicts the manufacturer's service proposal that maximizes its profits subject to delivering a certain level of profits to the retailer. The panel on the right depicts the profits the manufacturer and its rival will make under this proposal, if it is accepted by the retailer. When the manufacturer proposes a service that provides the same level of retailer profits as in the setting without competing proposals, i.e., a profit of $\xi_{R}$, then the manufacturer makes higher profits than its rival, i.e., $\xi_{C C}<\xi_{R M}$. Therefore, the rival has an incentive to come in with a proposal that offers higher profits for the retailer, and for itself. Thus, we have active competition for category captaincy, and the manufacturers bid up the retailer's profits. To this end, they propose a service that offers a higher service level and is also less biased. This continues up to

\footnotetext{
${ }^{11}$ Figure 3 illustrates the instance when $\theta=\frac{1}{2}$ and $k=\frac{1}{2}$. The curves in the left panel differ in units and scale. The curves in the right panel are in the same units and scale.
} 
the point where the manufacturers are indifferent between winning and losing category captaincy. In Figure 3, this corresponds to the point when retailer profits are $\Pi_{R}^{*}$.

So we find that, when the retailer can introduce active competition, it reduces the extent of bias in the category captain's service and induces a higher service level leading to higher profits for the retailer. However, inviting competing proposals does not always lead to active competition. As we will see later, in $\S 5$, this may motivate the retailer to consider engaging both manufacturers jointly when this is feasible.

\section{Manufacturer and Retailer Profits}

We now examine to what extent the manufacturers and the retailer gain from having the category captain arrangement, i.e., the impact on firm profits relative to the setting without the category captain arrangement.

Proposition 4. When there is active competition for category captaincy,

(i) the category captain and the rival manufacturer are better off than without the category captain arrangement if cross-price sensitivity is sufficiently high and the cost of providing a less-biased service is not too high $\left(k<\frac{257-16 \sqrt{229}}{33}\right.$ or, if $\frac{8}{9}>k \geq \frac{257-16 \sqrt{229}}{33}$ and $\theta>\theta_{4}$, where $\theta_{4}$ is defined in Appendix B). Otherwise, both are worse off.

(ii) the extent to which the retailer benefits from the arrangement decreases with cross-price sensitivity and with the cost of providing a less-biased service.

Proof. Refer Appendix B (pg 45).

Since active competition reduces the bias in the category captain's service, the scope for the rival manufacturer to benefit from under the category captain arrangement is higher when there is competition for category captaincy, i.e., the range of $\theta$ and $k$ for which the rival manufacturer is better off than without the category captain arrangement is higher than when only one particular manufacturer can offer service. This can be seen by comparing the hatched regions in Figures 1 and 2 .

By definition, the category captain's profits under active competition are lower than in the setting without competing proposals. But we find that the category captain can still benefit from the 
category captain arrangement. It follows from symmetry and the conditions for equilibrium (manufacturers are indifferent between winning and losing category captaincy) that both the category captain and rival manufacturer benefit from the arrangement in the same set of instances.

However, we find that active competition may also leave both manufacturers worse off than without the category captain arrangement. When $\theta$ is low or $k$ is high, active competition does not sufficiently reduce the bias in the service, because providing a less-biased service is not as valuable for a manufacturer when $\theta$ is low, and is more costly when $k$ is high. Since the service is relatively more biased, the rival manufacturer has a higher incentive to challenge the category captain. Consequently, the manufacturers compete away their profits by bidding increasing levels of a service that is too biased to be mutually beneficial.

From the retailer's perspective, while a more biased service results in a smaller boost to category demand, it also causes the manufacturers to compete more intensely for category captaincy and bid up the retailer's profits. We find that the latter aspect dominates as $\theta$ increases, whereas the former dominates as $k$ increases. Consequently, the extent to which the retailer benefits from the category captain arrangement decreases with $\theta$ and $k$.

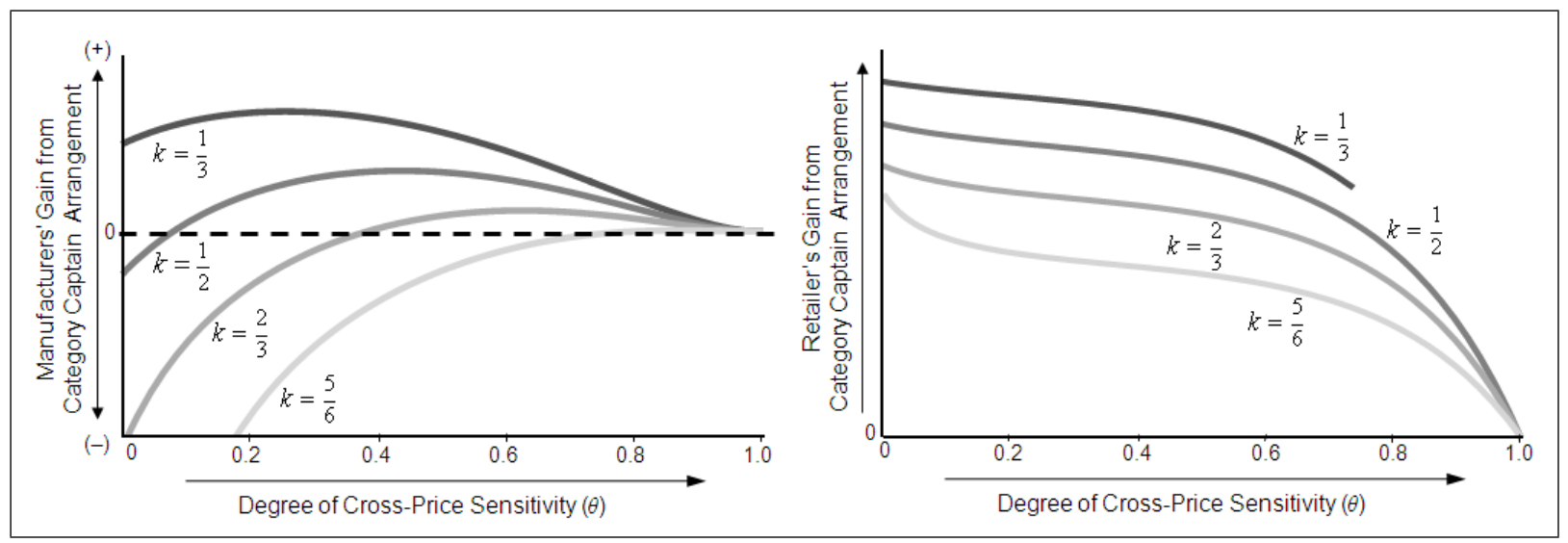

Figure 4: Manufacturers' and Retailer's Gain from Category Captain Arrangement ${ }^{12}$.

Thus, we find an interesting interaction between manufacturer price competition within a product category and their competition for category captaincy. Manufacturers are more likely to benefit from the category captain arrangement in product categories where the cross-price sensitivity between their brands is relatively high, since in this case the demand enhancing service helps soften

\footnotetext{
${ }^{12}$ For $k=\frac{1}{3}$, the graph for retailer's benefit stops at $\theta=0.73$ as beyond this point there are only equilibria without active competition.
} 
inter-brand competition. On the other hand, the retailer benefits to a larger extent in product categories where cross-price sensitivity between the brands is relatively low, since there is more intense competition amongst manufacturers for category captaincy. However, this competition might leave manufacturers worse off than before. Therefore, in such product categories, category captaincy may simply become the cost of doing business with the retailer. Figure 4 depicts the net benefit that manufacturers and the retailer gain from having the category captain arrangement.

\section{Category Captaincy When Joint Provision of Service is Feasible}

As an alternative to the category captain arrangement, a retailer may consider engaging both manufacturers simultaneously to provide retail service, provided it is feasible. In fact, if the relative efficiency of joint service provision is high, it would seem that this should be the retailer's preferred alternative, since it can tap the capabilities of both manufacturers rather than just one of them. We examine whether this is always the case; we investigate the conditions under which the category captain arrangement is preferred over joint service provision. We model the interactions in this setting as follows:

Stage 1 Proposal for category captaincy: $M_{1}, M_{2}$ simultaneously propose the service $\left(e_{i}, \rho_{i}\right)$ they would provide if selected as the category captain.

Stage 2 Category captaincy or Joint assignment decision: Retailer may accept one of their proposals, or may reject both and engage the manufacturers jointly, or may decide not to have any retail service. We denote the retailer's category captaincy decision by $r$ $\in\{0, J, 1,2\}$, where $r=i$ if $M_{i}$ 's proposal is accepted, $r=J$ if the retailer opts for joint assignment, and $r=0$ otherwise.

Stage 3 Service provision: If the retailer chose joint service provision, then the manufacturers simultaneously decide the service $\left(e_{i}^{J}, \rho_{i}^{J}\right)$ they will provide. If the retailer accepted $M_{i}$ 's proposal, then $M_{i}$ provides the service it proposed in Stage 1. Otherwise, there is no service provision.

Stage 4 Wholesale price decisions: Manufacturers simultaneously set their wholesale price $w_{i}$.

Stage 5 Retail price decision: Retailer sets retail prices $p_{i}$. 
In Stage 2, the retailer has the alternative of rejecting both category captaincy proposals and engaging the manufacturers jointly to provide service. If the retailer chose joint service provision, then, in Stage 3, the manufacturers simultaneously decide the service they will provide. Wholesale and retail price decisions then follow as before.

The retailer's profits are given by

$$
\Pi_{R}=\left(p_{1}-w_{1}\right) q_{1}+\left(p_{2}-w_{2}\right) q_{2} .
$$

$M_{1}$ 's and $M_{2}$ 's profits, respectively, are given by

$$
\begin{aligned}
& \Pi_{1}=w_{1} q_{1}-\delta(r=1) C\left(e_{1}, \rho_{1}\right)-\delta(r=J) \frac{1}{\mu} C\left(e_{1}^{J}, \rho_{1}^{J}\right), \\
& \Pi_{2}=w_{2} q_{2}-\delta(r=2) C\left(e_{2}, \rho_{2}\right)-\delta(r=J) \frac{1}{\mu} C\left(e_{2}^{J}, \rho_{2}^{J}\right),
\end{aligned}
$$

where $\delta(x)$ is an indicator function which equals one if $x$ is true and zero otherwise.

Let $\Pi_{R}^{k}$ and $\Pi_{i}^{k}$ be the equilibrium profits of the retailer and $M_{i}$, respectively, in the subgame at Stage 2 when the retailer has chosen the option $r=k, k \in\{0, J, 1,2\}$. Let $\left(e_{i}^{J *}, \rho_{i}^{J *}\right)$ denote the equilibrium service provided by $M_{i}$ under joint assignment in the subgame when the retailer has chosen joint service. Let $\Pi_{R}^{k *}$ and $\Pi_{i}^{k *}$ denote the subgame profits $\Pi_{R}^{k}$ and $\Pi_{i}^{k}$, respectively, in the subgame perfect equilibrium of the overall game. Let $\left(e_{i}^{*}, \rho_{i}^{*}\right)$ denote $M_{i}$ 's category captaincy proposal in an equilibrium of the overall game.

We solve for the subgame perfect Nash equilibrium of the above game. The details of the analysis are provided in Appendix C. Here, we only provide a brief description.

While in the earlier settings, a retailer engaged a category captain whenever a manufacturer proposed service (Lemma 1), this is longer the case when joint provision is feasible. Since joint service provision dominates not having any service, the retailer engages a category captain only when this arrangement outperforms joint service provision.

Whenever category captaincy occurs in equilibrium, we again define the notion of active competition by comparing the outcome to that in the setting without competing proposals, but where joint provision is feasible. Specifically, in any equilibrium where $M_{i}$ 's proposal is accepted with positive probability, we say that there is active competition for category captaincy if 


$$
\begin{aligned}
\Pi_{i}^{i *}<\max _{e_{i}, \rho_{i}} & \Pi_{i}^{i}, \\
& \text { s.t. } \quad \Pi_{R}^{i} \geq \max \left\{\Pi_{R}^{0 *}, \Pi_{R}^{J *}\right\} .
\end{aligned}
$$

We find that the three possible equilibrium outcomes - joint service provision, category captaincy without active competition and category captaincy with active competition - occur in mutually exclusive instances. We first discuss when the retailer may use joint service provision rather than the category captain arrangement.

Proposition 5. The retailer may employ joint service provision as an alternative to the category captain arrangement only if the relative efficiency of joint provision is sufficiently high $\left(\mu>\frac{1}{3}\right)$. Even when service provision under joint assignment is as efficient under category captaincy $(\mu=1)$, the retailer may still engage only one manufacturer as a category captain to provide service.

Proof. Refer Appendix B (pg 46).

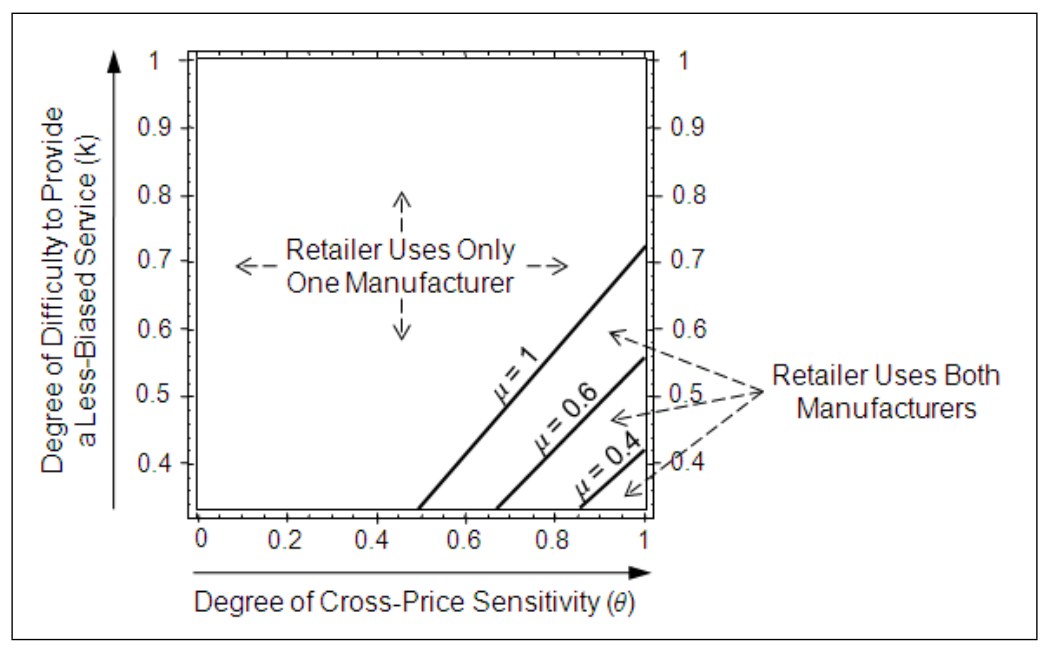

Figure 5: Category Captaincy vs. Joint Provision.

Figure 5 depicts the instances when the retailer engages only one manufacturer for varying levels of relative efficiency of joint service provision. Thus, interestingly, we find that even if the relative efficiency of joint service provision is high, the retailer may still engage only one of the manufacturers rather than tap the capabilities of both of them, since competition for category captaincy induces a service that provides higher profits for the retailer.

From the retailer's perspective, jointly engaging manufacturers can lead to better service provision either because competition for category captaincy is not sufficiently intense or because under 
joint service provision each manufacturer's service increases the other's incentive to provide service, i.e., there is a positive strategic interaction in service provision. In the former case, the manufacturers are worse off than if joint service provision was not feasible, whereas in the latter case, the manufacturers also benefit from joint service provision. We find that joint service provision occurs when cross-price sensitivity is sufficiently high and the cost of providing a less-biased service is sufficiently low. Of these instances, there is positive strategic interaction if each manufacturer's service under joint service provision is not too biased $\left(\rho_{i}^{J}>-\frac{\theta}{2}\right)$. In the remaining instances, joint service provision occurs because there is not sufficient competition for category captaincy between the manufacturers. Figure 6 depicts these instances when $\mu=1$.

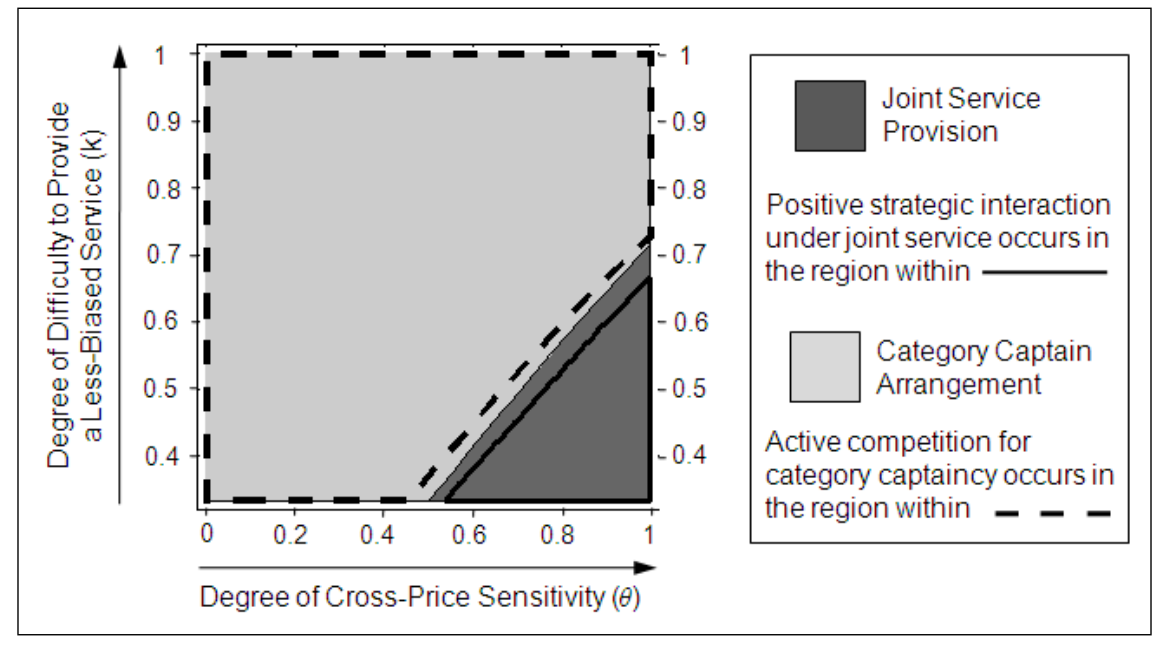

Figure 6: Category Captaincy When Joint Provision of Service is Feasible $(\mu=1)$.

However, as can be seen in Figure 6, we still do have category captaincy in equilibrium without active competition. In such instances, it is worthwhile for a manufacturer to propose a category captain arrangement that provides higher profits for the retailer than joint service provision. At the same time, this arrangement is sufficiently benevolent towards the rival that it is not worthwhile for the rival to challenge it. While there is no active competition, still the service provided is better (higher service level and lower bias) than when joint service provision was not feasible. In all other cases, there is active competition for category captaincy. The outcome in such instances is the same as when joint provision is not feasible; since competition for category captaincy is sufficient to drive up the retailer's profits, the joint service provision alternative does not have an impact. 


\section{Choice of the Category Captain}

We now extend the model to examine an issue that is likely to be of managerial interest, namely, what characteristics make one manufacturer a more likely candidate for category captaincy than its rival. To address this question, we examine the outcome when manufacturers differ along one of two possible dimensions: (i) their base level of demand, or (ii) their costs of providing a less-biased service. Since we are primarily interested in which manufacturer will be chosen as the category captain, we focus on the setting where joint service provision is not feasible. Table 2 summarizes our results. We discuss these in more detail below.

\begin{tabular}{|lc|}
\hline Manufacturers Differ in & Choice of Category Captain \\
\hline Base level of demand & Smaller manufacturer may become the category captain. \\
Service cost & More efficient manufacturer becomes the category captain. \\
\hline
\end{tabular}

Table 2: How Manufacturer Characteristics Influence Choice of Category Captain

\subsection{Asymmetry in Base Level of Demand}

Manufacturers may differ in their base level of demand due to underlying differences in consumer preferences, brand loyalty or product quality. We model this asymmetry in demand as follows:

$$
\begin{aligned}
& q_{1}=1+\alpha-\frac{\theta}{1-\theta}\left(p_{1}-p_{2}\right)-p_{1}, \\
& q_{2}=1-\alpha-\frac{\theta}{1-\theta}\left(p_{2}-p_{1}\right)-p_{2},
\end{aligned}
$$

where $M_{1}$ is taken to be the "larger" manufacturer with a higher base-level of demand, and $\alpha \in$ $\left[0, \frac{1}{2}\right]$ captures this asymmetry. Demand enhancing service by either manufacturer is taken to impact demand as before ${ }^{13}$.

Manufacturers make competing proposals and the sequence of interactions is as in $\S 4^{14}$. We restrict attention to instances where there is active competition. The details of the analysis can be found in the Technical Appendix (pg 57). We find that under active competition there is always a unique equilibrium.

\footnotetext{
${ }^{13}$ When $M_{i}$ provides service $\left(e_{i}, \rho_{i}\right)$, this has an own brand effect of $\frac{1}{2} e_{i}$ and a rival brand effect of $\frac{1}{2} \rho_{i} e_{i}$.

${ }^{14} \mathrm{We}$ assume that, when the retailer is indifferent between two proposals, then its choice is determined in equilibrium. To ensure uniqueness of equilibrium, we restrict attention to weakly dominant strategies.
} 
Interestingly, we find that, when all else is equal, the smaller manufacturer may, in fact, become the category captain. Figure 7 shows the instances where this occurs for various levels of asymmetry. In such instances, we find that the larger manufacturer may not have sufficient incentive to propose a service of low bias. This makes it imperative for the smaller manufacturer to become the category captain. In order for it to be attractive for the retailer to go with the smaller manufacturer, the smaller manufacturer proposes a service that is relatively less biased. Such a proposal also ensures that it is not worthwhile for the larger manufacturer to make an even more attractive proposal to the retailer ${ }^{15}$. As can be seen in Figure 7, we find that this is possible only when the cost of providing a less-biased service is not too high. When the asymmetry is higher, we find that there are fewer instances where the smaller manufacturer becomes the category captain.

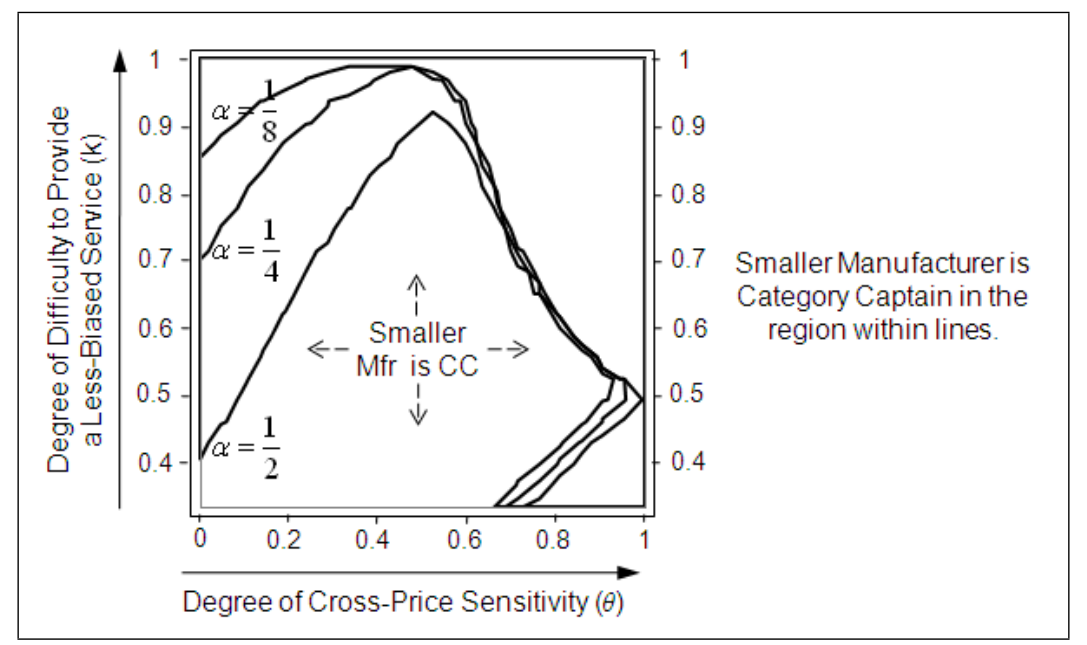

Figure 7: Choice of Category Captain with Asymmetric Base Level of Demand

Industry observers note that, while the category captain is usually the leading brand within the category, this is not always the case (e.g., ACNielsen 2005, Aftermarket Business 2003). A retailer may choose a smaller player if it believes the smaller player will be more objective than the leading brand. We find that this is indeed sensible.

\subsection{Asymmetry in Cost of Providing a Less-Biased Service}

Manufacturers are also likely to differ in their capabilities to provide demand enhancing service. Of particular interest is their ability to provide a less-biased service, since this is of primary concern

\footnotetext{
${ }^{15}$ There is still active competition for category captaincy in equilibrium. $M_{1}$ makes a proposal that is too biased, and $M_{2}$ 's proposal is driven by the threat of losing category captaincy to $M_{1}$. But $M_{2}$ 's proposal is sufficiently benevolent towards $M_{1}$ that it is not worthwhile for $M_{1}$ to make a more attractive proposal.
} 
to retailers. We consider that $M_{1}$ is more efficient than $M_{2}$ in providing a less-biased service. Let $k_{1}$ and $k_{2}$, respectively, be $M_{1}$ 's and $M_{2}$ 's degrees of difficulty to provide a less-biased service, with $k_{1}<k_{2}, k_{1}, k_{2} \in\left[\frac{1}{3}, 1\right)$.

Not surprisingly, we find that the more efficient manufacturer is always chosen as the category captain $^{16}$. Nevertheless, we find that the less-efficient manufacturer still benefits from improving its efficiency. By positioning itself as a more viable alternative, the less-efficient manufacturer can ensure that the category captain's service is less biased. This also benefits the retailer.

Thus, even if a manufacturer does not become the category captain, there is still value in acquiring this capability. Consultants often advice non-category captain manufacturers to invest in category management capabilities so that they can be category-captains-in-waiting and keep the current category captain in check. Our findings lend support to this advice.

\section{Conclusion}

Many retailers have come to rely on their category captains to make significant decisions regarding their in-store environment and offerings. Leading national manufacturers thus regard category captain assignments as an important part of their business strategy. But, even as such arrangements have emerged as the dominant mode of retail category management, there has been limited formal examination of this topic. Our research represents a step in this direction. We provide a modelbased analysis of the use of category captains for the provision of demand enhancing retailing service. We find that even when the category captain cares only about its own interests, it may still provide a service that also boosts the rival's demand. Moreover, the non-category manufacturer may benefit from the category captain arrangement even when the category captain's service depletes its demand. Both of these are more likely to occur in product categories where cross-price sensitivity between the competing brands is higher.

This leads to an interesting interaction between manufacturer price competition within a product category and their competition for category captaincy. In product categories where cross-price sensitivity between the brands is higher, there is lesser competition between manufacturers to become the category captain, since the category captain's service is relatively less-biased and the

\footnotetext{
${ }^{16} \mathrm{As}$ in $\S 6.1$, we take the interactions to be as in $\S 4$ and focus on instances with active competition.
} 
non-category captain manufacturer is more likely to benefit. On the other hand, in product categories where cross-price sensitivity between the brands is lower, the competition for category captaincy is more intense and retailer benefits to a larger extent. Manufacturers, however, may be worse off than without the category captain arrangement in such product categories. Furthermore, when competition for category captaincy is sufficiently intense, we find that it is worthwhile for the retailer to rely on only one manufacturer, even when it can tap the capabilities of both manufacturers to provide retail service. While we cannot comment on the impact of category captain arrangement on consumer welfare, we find that total category demand always increases.

We also find that a retailer may not always engage the leading national brand manufacturer as the category captain, since the smaller manufacturer may provide a less-biased service. Further, it is in the interest of a manufacturer to invest in its ability to provide a less-biased service even if it is not chosen as the category captain, since this keeps the current category captain in check, and ensures that its service is less-biased.

Our findings may help explain why, despite concerns regarding category captain opportunism, this practice of relying on only one manufacturer has flourished, and why there is limited evidence of harm to rival manufacturers. We now briefly discuss some of the limitations of our work and point to directions for future research.

We have focused on interactions at a single retailer. Expanding the scope of the analysis to include retail competition could shed light on additional issues of interest. Retail competition is likely to moderate the extent to which a retailer can increase its prices in response to demand enhancing service, which in turn could moderate the nature of strategic interaction between the manufacturers. Thus, one could examine how retail competition impacts the nature of service provided and the extent to which the manufacturers and retailers benefit from the category captain arrangement. On the other hand, if competing retailers engage category captains for demand enhancing service, and to the extent that the demand enhancing services are valued by consumers, both retailers may be able to raise prices. Further, the same manufacturers often vie for category captaincy at competing retailers. Thus, one could also examine the factors that lead competing retailers to choose either the same or different manufacturers as category captains and how this influences the outcomes.

We have assumed a linear demand and a quadratic-additive service cost formulation. We ac- 
knowledge this as a limitation of our approach. We conjecture that some of our results are likely to be relatively robust to alternate formulations. For $M_{2}$ to benefit even when $\rho_{1}<0$, all we require is the following: when retailing service has no impact on $M_{2}$ 's demand (i.e., $\rho_{1}=0$ ), the provision of such service enables $M_{1}$ to raise its wholesale price and this has a positive impact on $M_{2}$ 's demand. Then $M_{2}$ 's profits strictly increase for $\rho_{1}=0$, and, under mild regularity conditions, would increase for arbitrarily small $\rho_{1}<0$. Similarly, for $M_{1}$ to have an incentive to provide a less-biased service, we require that $M_{2}$ sets a higher wholesale price when $M_{1}$ 's service is less-biased than when it is more-biased, and that $M_{2}$ 's higher wholesale prices has a positive impact on $M_{1}$ 's demand. Further, in either case, the extent to which an increase in the wholesale price of one brand benefits the other is likely to increase with cross-price sensitivity. Nevertheless, future research could examine the scope for various outcomes under a more general demand and cost formulation.

Finally, while demand-enhancing activities are amongst the most common category captain responsibilities, there are other activities that a category captain may be asked to perform. In ongoing research, Wang, Raju and Dhar (2003) and Kurtulus and Toktay (2007) examine the consequences when a category captain and retailer collaborate on pricing under different sets of conditions. The category captain may also be responsible for sharing demand information with the retailer. Future research could explore these other activities when undertaken individually or in combination. 


\section{References}

ACNielsen. 2005. Consumer-centric Category Management. Published by John Wiley \& Sons, Inc. New Jersey.

Aftermarket Business. 2003. The Category Captain Steers the Ship. April 2003. 113(4). 8.

Arkader, R., C.F. Ferreira. Category Management Initiatives from the Retailer's Perspective: a Study in the Brazilian Grocery Industry. Journal of Purchasing and Supply Managament. 10(1). 41-51.

Basuroy, S., M. Mantrala and R.G. Walters. 2001. The Impact of Category Management on Retailer Prices and Performance: Theory and Evidence. Journal of Marketing. 65 (October) $16-32$.

Blattberg, R.C., E.J. Fox. 1995. Category Management: Getting Started, Guide 1. Washington, DC: Research Department, Food Marketing Institute.

Business Line. 2006. Growing Brands Together. May 2006. Available from http://www. thehindubusinessline.com/catalyst/2006/06/15/stories/2006061500020100.htm. Last accessed: May 2009.

Carameli, Jr., L.S. 2004. The Anti-competitive Effects and Antitrust Implications of Category Management and Category Captains of Consumer Products. Chicago-Kent Law Review. 79 $1315-1355$.

Cadbury Schweppes. 2005. 2005 Investor Seminar by President and CEO Gil Cassagne. Available from http://www.cadburyinvestors.com/cadbury_ir/res_press/. Last accessed: May 2009.

Campbell Soup. 2008. 2008 Investor Day Presentation. Webcast available from http:// investor.shareholder.com/campbell/webcasts.cfm. Last acessed: May 2009.

Cannondale Associates. 2005. Consumer Marketing At Retail: Category Management Benchmarking Study (Differentiation). Published by Cannondale Associates Inc.

Cannondale Associates. 2008. 2008 PoweRanking Results. Cannondale Associates Inc. press release. Available from http://www.cannondaleassoc.com/news_fs.htm. Last accessed: May 2009. 
Clorox. 2009. Company Presentation at the Consumer Analyst Group of New York Conference. Feb 2009. Available from http://investors.thecloroxcompany.com/events.cfm. Las accessed: May 2009.

Desrochers, D.M., G.T. Gundlach, A.A. Foer. 2003. Analysis of Antitrust Challenges to Category Captain Arrangements. Journal of Public Policy $\&$ Marketing. 22(2) 201-215.

ECR. 2005. The Case for ECR. A Review and Outlook for Continuous ECR Adoption in Western Europe. Available from www.ecrnet.org. Last acessed: May 2009.

ECR Conference. 2004. CategoryManagement is Here to Stay. Brussels. http://www.ecrnet.org/05projects/catman/Bxl\%202004_category\%20management.ppt. Last retrieved on Mar 23, 2008. FTC Report. 2001. Report on the Federal Trade Commission Workshop on Slotting Allowances and Other Marketing Practices in the Grocery Industry. Available at http://www.ftc.gov/os/2001/02/ slottingallowancesreportfinal.pdf. Last retrieved on Mar 23, 2008.

FTC Roundtable. 2003. Antitrust and Category Captains Roundtable Discussion. Available at http://antitrustinstitute.org/archives/files/270.pdf. Last retrieved on Mar 23, 2008.

Gruen, T.W. and R. Shah. 2000. Determinants and Outcomes of Plan and Objectivity and Implementation in Category Management Relationships. Journal of Retailing. 76(4) 483-510. Gajananan, S., S. Basuroy, S. Beldona. 2007. Category Management, Product Assortment and Consumer Welfare. Marketing Letters. 18 135-148.

Kurtulus M., L.B. Toktay. 2007. Category Captainship: Outsourcing Retail Category Management. Working Paper. Available at SSRN: http://ssrn.com/abstract=956577. Last accessed Mar 23, 2008.

Leary, T. B. 2004. A Second Look at Category Management. Available at http://www.ftc.gov/ speeches/leary/040519categorymgmt.pdf. Last retrieved on Mar 23, 2008.

Lindblom, A., R. Olkkonen. 2006. Nature And Effects Of Category Captain Arrangements in The Manufacturer-Retailer Relationships. The Finnish Journal Of Business Economics. 55(2) 181-193.

Freedman, M.P., M. Reyner, T. Tochtermann. 1997. European Category Management: Look Before You Leap. The McKinsey Quarterly. Issue 1. 156-164. 
Morgan, N. A., A. Kaleka, R. A. Gooner. 2007. Focal supplier opportunism in supermarket retailer category management. Journal of Operations Management 25 512-527.

NMR and AMA. 1992. Category Management: Positioning Your Organization to Win. NTC Publishing Group, 1992.

Progressive Grocer. 2003. Ties that Bind. Nov 2003. 100.

Progressive Grocer. 2006. Agents of Change. Nov 2006. 28 - 100.

Progressive Grocer. 2008. Marching Orders. Nov 2008. 87(12). 40-86.

Shubik, M. and R. Levitan. 1980. Market Structure and Behavior. Harvard University Press, Cambridge, MA.

Singh, A. and X. Vives. 1984. Price and Quantity Competition in a Differentiated Duopoly. Rand Journal of Economics. $15546 \mathbf{\square} 54$.

Steiner, R.L. 2001. Category Management - A Pervasive, New Vertical / Horizontal Format. Antitrust. 15(Spring). 77 - 81.

Supermarket News. 2003. The New Category Captain. June 2003.

Supermarket News. 2007. Managing Shelf Lives. Nov 2007. 55(45).

TRMM (Theory-Rich Marketing Modeling) Workshop. 2007. Keynote Address. Held in August 2007 at the Fuqua School of Business, Duke University.

Wang, Y., J. S. Raju, S. K. Dhar. 2003. The Choice and Consequences of Using a Category Captain for Category Management. Working Paper. Marketing Department, Wharton. University of Pennsylvania.

Wright, J. 2006. Antitrust Analysis Of Category Management: Conwood v.s. United States Tobacco Co. George Mason University Law and Economics Research Paper Series. Available from SSRN: http://ssrn.com/abstract_id=945178. Last retrieved on Mar 23, 2008.

Zenor, M.J. 1994. The Profit Benefits of Category Management. Journal of Marketing Research. 31 (May) 202-213. 


\section{Appendix A: Lemmas in Main Text}

\section{Proof for Lemma 1}

The subgame equilibrium profits when the retailer accepts and rejects $M_{1}$ 's proposal are given in Lemma C.1 in Appendix C (pg 48). It follows that $\Pi_{R}^{1}>\Pi_{R}^{0}$ whenever $e_{1}>0$, and $\Pi_{2}^{1}>\Pi_{2}^{0}$ iff $\rho_{1}>-\frac{\theta}{2}$. The total category demand is given by,

$$
q_{1}+q_{2}=\frac{4+e_{1}\left(1+\rho_{1}\right)}{4(2-\theta)}
$$

which strictly increases with $e_{1}$ whenever $\rho_{1}>-1$.

\section{Proof for Lemma 2.}

The subgame equilibrium profits when the retailer accepts and rejects $M_{1}$ 's proposal are given in Lemma C.1 in Appendix C (pg 48). Since the retailer accepts $M_{1}$ 's proposal whenever $e_{1}>0$, $M_{1}$ 's equilibrium proposal maximizes its profits $\Pi_{1}^{1}$. Thus we have,

$$
\frac{\partial \Pi_{1}^{1}}{\partial e_{1}}=0, \frac{\partial \Pi_{1}^{1}}{\partial \rho_{1}}=0 \Longrightarrow e_{1}=\frac{2 k(2-\theta)(1-\theta)(2+\theta)}{4 k\left(15+2 \theta-9 \theta^{2}+\theta^{4}\right)-\theta^{2}(1-\theta)}, \rho_{1}=\frac{\theta-2 k}{k(2-\theta)}
$$

The equilibrium profits are given by,

$$
\begin{aligned}
\Pi_{1}^{*}= & \frac{2 k(1-\theta)(2+\theta)^{2}}{4 k\left(15+2 \theta-9 \theta^{2}+\theta^{4}\right)-\theta^{2}(1-\theta)}, \\
\Pi_{2}^{*}= & \frac{(1-\theta)\left[\theta(1-\theta)+k\left(28+21 \theta-9 \theta^{2}-4 \theta^{3}\right)\right]^{2}}{2\left[4 k\left(15+2 \theta-9 \theta^{2}+\theta^{4}\right)-\theta^{2}(1-\theta)\right]^{2}}, \\
\Pi_{R}^{*}= & \frac{\theta^{2}(1-\theta)^{2}+2 k \theta\left(28+25 \theta-46 \theta^{2}-19 \theta^{3}+8 \theta^{4}+4 \theta^{5}\right)}{4(1+\theta)\left[4 k\left(15+2 \theta-9 \theta^{2}+\theta^{4}\right)-\theta^{2}(1-\theta)\right]^{2}} \\
& -\frac{k^{2}\left(1808+3992 \theta+1921 \theta^{2}-1466 \theta^{3}-1255 \theta^{4}-16 \theta^{5}+168 \theta^{6}+32 \theta^{7}\right)}{4(1+\theta)\left[4 k\left(15+2 \theta-9 \theta^{2}+\theta^{4}\right)-\theta^{2}(1-\theta)\right]^{2}} .
\end{aligned}
$$

\section{Proof for Lemma 3}

Lemma C.2 in Appendix C (pg 49) provides the equilibrium profits in the subgames at Stage 3. The conditions for equilibrium in (15) to (18) are not easy to work with since $\Omega_{i}$ and $\Omega_{j}$ are possibly discontinuous when $\Pi_{R}^{i}=\Pi_{R}^{j}$. However, as we show in Lemma C.3 in Appendix C (pg 49), this discontinuity does not matter for the purpose of our analysis; by adding condition (C.7) (refer 
Lemma C.3), we can essentially replace $\Omega_{i}$ and $\Omega_{j}$ with $\Pi_{i}^{i}$ and $\Pi_{j}^{i}$, respectively, in the equilibrium conditions. Therefore, we work with the equilibrium conditions described in Lemma C.3.

We now consider an equilibrium without active competition, where $M_{i}$ is chosen as the category captain with positive probability. From (19) we have,

$$
\begin{aligned}
\Pi_{i}^{i *}= & \max _{e_{i}, \rho_{i}} \Pi_{i}^{i}, \\
& \text { s.t. } \quad \Pi_{R}^{i} \geq \Pi_{R}^{0 *} .
\end{aligned}
$$

Since the manufacturers are symmetric, we know from Lemma 2 that the programming problem above has a unique optimum. Therefore, there is a unique $\left(e_{i}^{*}, \rho_{i}^{*}\right)$ that satisfies (A.4) and is, in fact, the same as the equilibrium proposal in the setting where only $M_{i}$ can offer service. In other words, from Lemma 2,

$$
e_{i}^{*}=\frac{2 k(2-\theta)(1-\theta)(2+\theta)}{4 k\left(15+2 \theta-9 \theta^{2}+\theta^{4}\right)-\theta^{2}(1-\theta)}, \rho_{i}^{*}=\frac{\theta-2 k}{k(2-\theta)},
$$

and the corresponding profits are,

$$
\begin{aligned}
\Pi_{i}^{i *}=\xi_{C C} & =\frac{2 k(1-\theta)(2+\theta)^{2}}{4 k\left(15+2 \theta-9 \theta^{2}+\theta^{4}\right)-\theta^{2}(1-\theta)}, \\
\Pi_{j}^{i *}=\xi_{R M} & =\frac{(1-\theta)\left[\theta(1-\theta)+k\left(28+21 \theta-9 \theta^{2}-4 \theta^{3}\right)\right]^{2}}{2\left[4 k\left(15+2 \theta-9 \theta^{2}+\theta^{4}\right)-\theta^{2}(1-\theta)\right]^{2}} \\
\Pi_{R}^{i *}=\xi_{R} & =\frac{\theta^{2}(1-\theta)^{2}+2 k \theta\left(28+25 \theta-46 \theta^{2}-19 \theta^{3}+8 \theta^{4}+4 \theta^{5}\right)}{4(1+\theta)\left[4 k\left(15+2 \theta-9 \theta^{2}+\theta^{4}\right)-\theta^{2}(1-\theta)\right]^{2}} \\
& -\frac{k^{2}\left(1808+3992 \theta+1921 \theta^{2}-1466 \theta^{3}-1255 \theta^{4}-16 \theta^{5}+168 \theta^{6}+32 \theta^{7}\right)}{4(1+\theta)\left[4 k\left(15+2 \theta-9 \theta^{2}+\theta^{4}\right)-\theta^{2}(1-\theta)\right]^{2}}
\end{aligned}
$$

It also follows from Lemma 2 and Lemma C.2 that,

$$
\Pi_{R}^{i *}=\xi_{R}>\Pi_{R}^{0 *}
$$

We now apply the equilibrium conditions in Lemma C.3. We have from (C.8), (A.8) and (A.9),

$$
\xi_{R} \geq \Pi_{R}^{j *}
$$


From (C.9) and (A.6),

$$
\xi_{C C} \geq \Pi_{i}^{j *}
$$

From (C.11), (A.7) and (A.9),

$$
\begin{aligned}
& \xi_{R M} \geq \max _{e_{j}, \rho_{j}} \Pi_{j}^{j}, \\
& \text { s.t. } \quad \Pi_{R}^{j} \geq \xi_{R} .
\end{aligned}
$$

But the solution to the programming problem on the right hand side is the same as when only $M_{j}$ can make a proposal, since $\Pi_{R}^{j}=\xi_{R}$ in that case. Thus we have,

$$
\xi_{R M} \geq \xi_{C C}
$$

Finally, if $\Pi_{R}^{i *}=\Pi_{R}^{j *}=\xi_{R}$, then, since $M_{j}$ is chosen with positive probability, we have from (C.10) and (A.9),

$$
\begin{gathered}
\Pi_{j}^{j *}=\max _{e_{j}, \rho_{j}} \Pi_{j}^{j}=\xi_{C C} . \\
\text { s.t. } \Pi_{R}^{j} \geq \xi_{R} .
\end{gathered}
$$

Then from (C.7), (A.6) and (A.7), we have,

$$
\Pi_{R}^{i *}=\Pi_{R}^{j *} \Longrightarrow \xi_{C C}=\xi_{R M}
$$

Thus, the necessary and sufficient conditions for equilibria without active competition are given by (A.5) and (A.10), (A.11), (A.13) and (A.15). In particular we require that $\xi_{R M} \geq \xi_{C C}$, and $M_{i}$ 's proposal is unique. However, $M_{j}$ 's is not; any proposal that satisfies (A.10) and (A.11) is admissible. This leads to degeneracy. In particular, $e_{2}=0$ satisfies both (A.10) and (A.11). Thus, whenever $\xi_{R M} \geq \xi_{C C}$, we have equilibria without active competition.

We now consider equilibria with active competition. From (C.10) and (19), we have,

$$
\begin{aligned}
& \max _{e_{i}, \rho_{i}} \quad \Pi_{i}^{i} \quad<\max _{e_{i}, \rho_{i}} \Pi_{i}^{i}, \\
& \text { s.t. } \quad \Pi_{R}^{i} \geq \max \left\{\Pi_{R}^{0 *}, \Pi_{R}^{j *}\right\} \quad \text { s.t. } \quad \Pi_{R}^{i} \geq \Pi_{R}^{0 *} \text {. }
\end{aligned}
$$

The programming problem on the left hand side differs from the one on the right hand side in that it has one additional constraint. Thus, this constraint must be more binding and therefore, we 
have,

$$
\Pi_{R}^{i *}=\Pi_{R}^{j *}>\Pi_{R}^{0 *}
$$

Therefore, in any equilibrium with active competition, the retailer chooses either manufacturer with equal probability. Further, from (A.17) and since the manufacturers are symmetric, we have,

$$
\begin{aligned}
& \Pi_{i}^{i *}=\max _{e_{i}, \rho_{i}} \Pi_{i}^{i} \quad=\max _{e_{j}, \rho_{j}} \Pi_{j}^{j} \quad=\Pi_{j}^{j *}, \\
& \text { s.t. } \Pi_{R}^{i} \geq \max \left\{\Pi_{R}^{0 *}, \Pi_{R}^{j *}\right\} \quad \text { s.t. } \Pi_{R}^{j} \geq \max \left\{\Pi_{R}^{0 *}, \Pi_{R}^{i *}\right\} \text {. }
\end{aligned}
$$

Then, from (A.17), (C.7) and (A.18), we have,

$$
\Pi_{i}^{i *}=\Pi_{i}^{j *}=\Pi_{j}^{j *}=\Pi_{j}^{i *}
$$

Consider $M_{i}$ 's proposal. From (A.19), we have,

$$
\begin{aligned}
\Pi_{i}^{i *} & =\Pi_{j}^{i *}, \\
\Longrightarrow \quad e_{i}^{*} & =\frac{4(1-k)(1-\theta)\left(1-\rho_{1}\right)}{15+k(1-\theta)+\theta(1-4 \theta)+8 k\left(4-\theta^{2}\right) \rho_{i}^{*}+\left(1-\theta+k\left(15+\theta-4 \theta^{2}\right)\right) \rho_{i}^{* 2}} .
\end{aligned}
$$

Also, from (C.7) and (A.17) we have,

$$
\begin{aligned}
\left(e_{i}^{*}, \rho_{i}^{*}\right) \in \max _{e_{i}, \rho_{i}} & \Pi_{i}^{i}, \\
& \text { s.t. } \quad \Pi_{R}^{i}=\Pi_{R}^{j *} .
\end{aligned}
$$

From the Kuhn-Tucker first order conditions, we have ${ }^{17}$,

$$
\frac{\partial \Pi_{i}^{i}}{\partial e_{i}}-\lambda \frac{\partial \Pi_{R}^{i}}{\partial e_{i}}=0, \frac{\partial \Pi_{i}^{i}}{\partial \rho_{i}}-\lambda \frac{\partial \Pi_{R}^{i}}{\partial \rho_{i}}=0, \lambda>0
$$

where $\lambda$ is the Lagrangian multiplier. Since, from Lemma C.2, we know that $\Pi_{R}^{i}$ is strictly increasing

\footnotetext{
${ }^{17}$ Since the objective function is continuous and constraint set is compact, a maximum exists. Further, the KuhnTucker conditions are necessary as constraint qualification is satisfied. Specifically, it can be seen from the results in Lemma C.2 that $\frac{\partial \Pi_{R}^{i}}{\partial e_{i}}>0$ and $\frac{\partial \Pi_{R}^{i}}{\partial \rho_{i}}>0$.
} 
in $e_{i}$ and $\rho_{i}$, the above conditions reduce to,

$$
\begin{aligned}
-\frac{\frac{\partial \Pi_{i}^{i}}{\partial e_{i}}}{\frac{\partial \Pi_{R}^{i}}{\partial e_{i}}} & =-\frac{\frac{\partial \Pi_{i}^{i}}{\partial \rho_{i}}}{\frac{\partial \Pi_{R}^{i}}{\partial \rho_{i}}} \\
\frac{\partial \Pi_{i}^{i}}{\partial e_{i}} & <0 .
\end{aligned}
$$

(A.20), (A.24) and (A.25) constitute necessary conditions that $M_{i}$ 's proposal must satisfy, and are sufficient along with (A.17), (A.18) and (A.19) for an equilibrium. We use these to establish that an equilibrium with active competition is unique when it exists and exists iff $\xi_{C C}>\xi_{R M}$.

Substituting from (A.21) in (A.24) we have,

$$
\begin{aligned}
& {\left[(1-\theta)^{3}+2 k\left(31-77 \theta+21 \theta^{2}+29 \theta^{3}-4 \theta^{4}\right)-\right.} \\
& \left.k^{2}\left(319+611 \theta+941 \theta^{2}+569 \theta^{3}+136 \theta^{4}+16 \theta^{5}\right)\right] \rho_{i}^{* 4} \\
+\quad & {\left[4(1-\theta)^{2}\left(7+\theta-2 \theta^{2}\right)-2 k\left(87+147 \theta+239 \theta^{2}+141 \theta^{3}+30 \theta^{4}+4 \theta^{5}\right)-\right.} \\
& \left.k^{2}\left(89+461 \theta+421 \theta^{2}+235 \theta^{3}+82 \theta^{4}+8 \theta^{5}\right)\right] \rho_{i}^{* 3} \\
-\quad & 2\left[45-71 \theta+27 \theta^{2}+15 \theta^{3}-16 \theta^{4}+2 k\left(211+823 \theta+869 \theta^{2}+509 \theta^{3}+164 \theta^{4}+16 \theta^{5}\right)+\right. \\
& \left.k^{2}\left(301+729 \theta+923 \theta^{2}+503 \theta^{3}+120 \theta^{4}+16 \theta^{5}\right)\right] \rho_{i}^{* 2} \\
-\quad & 4\left[105+413 \theta+433 \theta^{2}+255 \theta^{3}+82 \theta^{4}+8 \theta^{5}-2 k\left(57+189 \theta+221 \theta^{2}+135 \theta^{3}+42 \theta^{4}+4 \theta^{5}\right)+\right. \\
& \left.k^{2}\left(265+733 \theta+905 \theta^{2}+527 \theta^{3}+146 \theta^{4}+16 \theta^{5}\right)\right] \rho_{i}^{*} \\
+\quad 225-797 \theta-875 \theta^{2}-519 \theta^{3}-160 \theta^{4}-16 \theta^{5}-2 k\left(257+749 \theta+899 \theta^{2}+523 \theta^{3}+148 \theta^{4}+16 \theta^{5}\right)+ & (\text { A.26) } \\
& k^{2}(1-\theta)^{2}\left(33-\theta-8 \theta^{2}\right)=0 .
\end{aligned}
$$

For $\rho_{i}^{*}=-1$, the left hand side reduces to,

$$
48(1-k)^{2}\left(11+55 \theta+53 \theta^{2}+31 \theta^{3}+11 \theta^{4}+\theta^{5}\right)>0
$$

and for $\rho_{i}^{*}=1$, it reduces to,

$$
-16(1+3 k)^{2}(1+\theta)(2+\theta)^{4}<0 .
$$


Thus, the quartic equation has either one or three roots in $[-1,1]$. It is tedious, but straight forward to show using the Descartes sign change rule that, in fact, it has only one root in $[-1,1]$ for all $\theta, k^{18}$. This establishes uniqueness of the $M_{i}$ 's proposal in an equilibrium. Since $M_{j}$ 's proposal must also satisfy the same conditions, it follows that,

$$
e_{j}^{*}=e_{i}^{*} \text { and } \rho_{j}^{*}=\rho_{i}^{*} .
$$

Thus, the equilibrium is unique. Further, (A.17), (A.18) and (A.19) are also satisfied. Therefore, an equilibrium exists iff the solution to (A.26) also satisfies (A.25).

The boundary conditions for the set of instances (i.e., the set of $(\theta, k))$ for which an equilibrium exists can be obtained from (A.20), (A.24) and (A.25), when (A.25) just fails to hold. In other words, teh boundary conditions are given by

$$
\begin{gathered}
\Pi_{i}^{i *}=\Pi_{j}^{i *},-\frac{\frac{\partial \Pi_{i}^{i}}{\partial e_{i}}}{\frac{\partial \Pi_{R}^{i}}{\partial e_{i}}}=-\frac{\frac{\partial \Pi_{i}^{i}}{\partial \rho_{i}}}{\frac{\partial \Pi_{R}^{i}}{\partial \rho_{i}}}, \frac{\partial \Pi_{i}^{i}}{\partial e_{i}}=0, \\
\Longrightarrow \quad \Pi_{i}^{i *}=\Pi_{j}^{i *}, \frac{\partial \Pi_{i}^{i}}{\partial e_{i}}=0, \frac{\partial \Pi_{i}^{i}}{\partial \rho_{i}}=0 .
\end{gathered}
$$

But these conditions corresponds to the instance where $M_{i}$ makes a proposal that maximizes its profits and $M_{i}$ and $M_{j}$ realize the same profits, which is the same instance as wehn $\xi_{C C}=\xi_{R M}$. Thus, the set of instances when active competition occurs has the same boundary as the set of instances without active competition. Hence, they either coincide or are mutually exclusive and exhaustive. To establish which of this is true, we proceed numerically. We consider an arbitrary instance $(\theta, k)=\left(\frac{1}{2}, \frac{1}{2}\right)$. It is straightforward to show that in this case $\xi_{C C}>\xi_{R M}$. Further, (A.26) reduces to,

$$
7479 \rho_{i}^{* 4}+45184 \rho_{i}^{* 3}+75074 \rho_{i}^{* 2}+59384 \rho_{i}^{*}+379=0
$$

and it can be verified that the solution satisfies (A.25).

\footnotetext{
${ }^{18}$ In the quartic equation, the coefficients of all powers of $\rho_{i}^{*}$ are negative and the constant term may either be negative or positive. Taking the second derivative (w.r.t. $\rho_{i}^{*}$ ) of the quartic polynomial and applying the Descartes sign change rule, it follows that the left hand side has at most one local maxima (and one local minima) for $\rho_{i}^{*}<0$ and is strictly increasing for $\rho_{i}^{*}>0$. This is possible only when there is one root in $[-1,1]$.
} 


\section{Appendix B: Propositions}

\section{Proof for Proposition 1}

From Lemma 1, we know that the retailer accepts the proposal whenever $e_{1}>0$ and is better off. $M_{1}$ is better off since the equilibrium proposal maximizes its profits. From Lemma 2, we have

$$
\rho_{1}>0 \Longrightarrow k<\frac{\theta}{2}
$$

Since $k \geq \frac{1}{3}$, we have $\theta>\frac{2}{3}$.

For $M_{2}$ to be better off, we require from Lemmas 1 and 2 that

$$
\rho_{1}>-\frac{\theta}{2} \Longrightarrow \theta>4-2 \sqrt{3}, k<\frac{2 \theta}{4-2 \theta+\theta^{2}}
$$

\section{Proof for Proposition 2}

From Lemma 3, we have equilibrium with active competition when $\xi_{C C}>\xi_{R M}$. From (A.6) and (A.7), we have

$$
\Longrightarrow k^{2}\left(176+88 \theta-57 \theta^{2}+\theta^{3}+8 \theta^{4}\right)-2 k \theta(4-\theta)(1+\theta)(7+2 \theta)-(1-\theta) \theta^{2}>0 .
$$

For $k=1$, the left hand side reduces to,

$$
4(2-\theta)^{2}\left(11+13 \theta+3 \theta^{2}\right)
$$

which is always positive, and for $k=\frac{1}{3}$, it reduces to,

$$
\frac{4}{9}(1-\theta)^{2}\left(44-20 \theta-60 \theta^{2}+4 \theta^{3}+5 \theta^{4}\right)
$$

If the above expression is also positive, then we have an equilibrium with active competition for all $k$, whereas if it is negative, then we have such an equilibrium only when $k>k_{3}$, where,

$$
k_{3}=\frac{\theta\left(28+29 \theta-\theta^{2}-2 \theta^{3}\right)+\theta(2+\theta) \sqrt{(2+\theta)\left(30+3 \theta-7 \theta^{2}+\theta^{3}\right)}}{176+88 \theta-57 \theta^{2}+\theta^{3}+8 \theta^{4}} .
$$


(B.5) is positive if,

$$
44-20 \theta-60 \theta^{2}+4 \theta^{3}+5 \theta^{4}>0
$$

The left hand side is positive for $\theta=0$, negative for $\theta=1$, and has at most one positive root by Descartes sign change rule. Solving numerically, we find that the root is $\theta_{3} \approx 0.73$. Then, for $\theta<\theta_{3}$, we have equilibrium with active competition for all $k$ and for $\theta \geq \theta_{3}$, we have active competition for $k>k_{3}$.

\section{Proof for Proposition 3}

We know from Lemma 2, that in the setting of $\S 3$, the equilibrium service proposal is given by

$$
e_{i}=\frac{2 k(2-\theta)(1-\theta)(2+\theta)}{4 k\left(15+2 \theta-9 \theta^{2}+\theta^{4}\right)-\theta^{2}(1-\theta)}, \rho_{i}=\frac{\theta-2 k}{k(2-\theta)}
$$

Consider the condition (A.26) that $\rho_{i}^{*}$ must satisfy in any equilibrium with active competition. We know from (A.27) and that the left handside is positive for $\rho_{i}=-1$. Substituting for $\rho_{i}=\frac{\theta-2 k}{k(2-\theta)}$ from (B.8) in the left hand side of (A.26), we have,

$$
\begin{aligned}
& \frac{(1-k)^{2}}{k^{4}(2-\theta)^{4}}\left[k^{2}\left(176+88 \theta-57 \theta^{2}+\theta^{3}+8 \theta^{4}\right)-2 k \theta(1+\theta)(4-\theta)(7+2 \theta)-\theta^{2}(1-\theta)\right] \\
& {\left[\theta^{2}(1-\theta)+2 k \theta\left(4-\theta+22 \theta^{2}+9 \theta^{3}+2 \theta^{4}\right)+k^{2}(1-\theta)\left(48+152 \theta+71 \theta^{2}+17 \theta^{3}\right)\right] .}
\end{aligned}
$$

We know from (B.3) the second term in the above expression is strictly positive when there is active competition. Thus, the entire expression is positive when there is active competition. In other words, $\rho_{i}^{*}>\rho_{i}=\frac{\theta-2 k}{k(2-\theta)}$.

We verify by solving (A.26) and (A.21) numerically that the service level is higher than in the setting without competition for category captaincy.

\section{Proof for Proposition 4}

We know from (A.20) that when there is active competition, the category captain and the non-category captain manufacturer make the same profits. Further, we know from Lemma 1 that

the rival manufacturer is worse off if $\rho_{i}^{*}<-\frac{\theta}{2}$. Consider the condition (A.26) that $\rho_{i}^{*}$ must satisfy in any equilibrium with active competition. We know from (A.27) and that the left handside is 
positive for $\rho_{i}=-1$. Substituting for $\rho_{i}=-\frac{\theta}{2}$ in the left hand side of (A.26), we have,

$$
\begin{aligned}
& \frac{(2+\theta)^{4}}{16}\left[\quad 225+557 \theta+227 \theta^{2}+15 \theta^{3}-k\left(514+698 \theta+598 \theta^{2}+166 \theta^{3}-56 \theta^{4}\right)+\right. \\
& \left.k^{2}\left(33+397 \theta+499 \theta^{2}-105 \theta^{3}+56 \theta^{4}-16 \theta^{5}\right)\right] .
\end{aligned}
$$

If the above expression is positive then $\rho_{i}^{*}>-\frac{\theta}{2}$. The expression is quadratic in $k$, positive for $k=\frac{1}{3}$ as it reduces to

$$
\frac{(2+\theta)^{4}}{36}\left(129+829 \theta+187 \theta^{2}-117 \theta^{3}+56 \theta^{4}-4 \theta^{5}\right)
$$

and negative for $k=1$ as it reduces to $-\left(4-\theta^{2}\right)^{4}$. It is tedious but straightforward to show that (B.10) is positive if

$$
k<\frac{257-16 \sqrt{229}}{33} \text { or } \frac{8}{9}>k \geq \frac{257-16 \sqrt{229}}{33} \text { and } \theta>\theta_{4},
$$

where $\theta_{4}$ is the root of the equation

$$
\begin{aligned}
& 225+557 \theta+227 \theta^{2}+15 \theta^{3}-k\left(514+698 \theta+598 \theta^{2}+166 \theta^{3}-56 \theta^{4}\right) \\
& +\quad k^{2}\left(33+397 \theta+499 \theta^{2}-105 \theta^{3}+56 \theta^{4}-16 \theta^{5}\right)=0,
\end{aligned}
$$

in $(0,1)^{19}$.

We verify numerically that the extent to which the retailer benefits from the arrangement decreases with $\theta$ and $k$. Figure 4 depicts the result graphically.

\section{Proof for Proposition 5}

The profits in the subgame starting at stage 3 when the retailer chooses not to have service is as before. Lemma C.4 provides the profits in the subgame starting at Stage 3 when the retailer chooses joint service provision. It is straightforward to verify that

$$
\Pi_{R}^{J *}>\Pi_{R}^{0}
$$

\footnotetext{
${ }^{19}$ This can also be verified using Mathematica.
} 
Therefore, the joint service provision alternative dominates not having any service. The retailer chooses joint service provision only if the category captaincy proposals do not provide better profits than joint service provision. Thus, it follows that we have joint service provision in equilibrium iff neither manufacturer can make a proposal that offers better profits for itself and the retailer, i.e., iff,

$$
\nexists\left(e_{i}, \rho_{i}\right) \text { s.t. } \Pi_{R}^{i} \geq \Pi_{R}^{J *} \text { and } \Pi_{i}^{i} \geq \Pi_{i}^{J *}, i \in\{1,2\}
$$

For otherwise, $M_{i}$ would have an incentive make such a proposal and joint service provision cannot occur in equilibrium.

When (B.15) does not hold, let $\xi_{C C}^{\prime}, \xi_{R M}^{\prime}$ and $\xi_{R}^{\prime}$ be the profits of the category captain, the rival manufacturer and the retailer, respectively, in the absence of competition for category captaincy. These are given by,

$$
\begin{aligned}
& \xi_{C C}^{\prime}=\Pi_{1}^{1}\left(e_{1}^{\prime}, \rho_{1}^{\prime}\right), \xi_{C C}^{\prime}=\Pi_{2}^{1}\left(e_{1}^{\prime}, \rho_{1}^{\prime}\right), \xi_{R}^{\prime}=\Pi_{R}^{1}\left(e_{1}^{\prime}, \rho_{1}^{\prime}\right), \\
& \text { where }\left(e_{1}^{\prime}, \rho_{1}^{\prime}\right) \text { s.t. }\left(e_{1}^{\prime}, \rho_{1}^{\prime}\right) \in \underset{e_{1}, \rho_{1}}{\operatorname{argmax}} \Pi_{1}^{1}, \\
& \text { s.t. } \Pi_{R}^{1} \geq \Pi_{R}^{J *} .
\end{aligned}
$$

As before, an equilibrium with category captaincy but without active competition can occur iff

$$
\xi_{C C}^{\prime} \leq \xi_{R M}^{\prime}
$$

Lastly, in an equilibrium with active competition for category captaincy, the category captain's proposal is driven by the threat of losing category captaincy to its rival, as before it follows that $\Pi_{R}^{i *}=\Pi_{R}^{j *}$. Of course, this must be more attractive for the retailer than joint service provision. Therefore, for such an equilibrium, we require that,

$$
\Pi_{R}^{i *}=\Pi_{R}^{j *} \geq \Pi_{R}^{J *}
$$

The rest of the conditions are as in the case when joint service provision was not feasible since, as can be seen from (B.18), the joint service provision is now not relevant for the retailer.

It follows from (B.15) that an equilibrium without active competition for category captaincy 
and an equilibrium with joint service provision occur in mutually exclusive cases. Further, by numerically verifying when (B.18) holds, we find that an equilibrium with active competition for category captaincy occurs iff neither (B.15) nor (B.17) holds. It can also be shown that (B.15) holds for some $\theta, k$ iff $\mu>\frac{1}{3}$. Finally, we verify numerically that (B.15) does not hold for all $\theta, k$ when $\mu=1$.

\section{Appendix C: Additional Lemmas}

Lemma C.1. For the setting in $\S 3$, in the subgame where the retailer rejects $M_{1}$ 's proposal, equilibrium profits are given by

$$
\Pi_{R}^{0}=\frac{1}{2(2-\theta)^{2}}, \Pi_{1}^{0}=\Pi_{2}^{0}=\frac{1-\theta}{2(2-\theta)^{2}}
$$

In the subgame where the retailer accepts $M_{1}$ 's proposal, equilibrium profits are given by

$$
\begin{aligned}
\Pi_{R}^{1} & =\frac{1}{2(2-\theta)^{2}}+\frac{1+\rho_{1}}{4(2-\theta)^{2}} e_{1}+\frac{\left(4+5 \theta^{2}\right)\left(1+\rho_{1}^{2}\right)+2 \theta\left(8+\theta^{2}\right) \rho_{1}}{16(1+\theta)\left(4-\theta^{2}\right)^{2}} e_{1}^{2}, \\
\Pi_{1}^{1} & =\frac{(1-\theta)\left(2(2+\theta)+\left(2+\theta \rho_{1}\right) e_{1}\right)^{2}}{8\left(4-\theta^{2}\right)^{2}}-\frac{1}{2}\left(1+\frac{k}{1-k}\left(1+\rho_{1}\right)^{2}\right) e_{1}^{2}, \\
\Pi_{2}^{1} & =\frac{(1-\theta)\left(2(2+\theta)+\left(\theta+2 \rho_{1}\right) e_{1}\right)^{2}}{8\left(4-\theta^{2}\right)^{2}} .
\end{aligned}
$$

Consider the subgame where the retailer has accepted $M_{1}$ 's proposal. Solving for the equilibrium wholesale prices and retail prices for this subgame in the usual manner we have

$$
\begin{aligned}
& w_{1}=\frac{(1-\theta)\left(2(2+\theta)+\left(2+\theta \rho_{1}\right) e_{1}\right)}{2\left(4-\theta^{2}\right)}, \\
& w_{2}=\frac{(1-\theta)\left(2(2+\theta)+\left(\theta+2 \rho_{1}\right) e_{1}\right)}{2\left(4-\theta^{2}\right)}, \\
& p_{1}=\frac{3-2 \theta}{2(2-\theta)}+\frac{3\left(2-\theta^{2}\right)+\left(5-2 \theta^{2}\right) \theta \rho_{1}}{4(1+\theta)\left(4-\theta^{2}\right)} e_{1}, \\
& p_{2}=\frac{3-2 \theta}{2(2-\theta)}+\frac{\left(5-2 \theta^{2}\right) \theta+3\left(2-\theta^{2}\right) \rho_{1}}{4(1+\theta)\left(4-\theta^{2}\right)} e_{1} .
\end{aligned}
$$

The equilibrium profits then are as stated in the Lemma.

For the subgame where the retailer has rejected $M_{1}$ 's proposal, we can obtain the equilibrium 
strategies by substituting $e_{1}=0$ in (C.3) to (C.6). The equilibrium profits are as stated in the Lemma.

Lemma C.2. For the setting in $\S 4$, in the subgame where the retailer rejects both proposals, equilibrium profits are given by

$$
\Pi_{R}^{0}=\frac{1}{2(2-\theta)^{2}}, \Pi_{1}^{0}=\Pi_{2}^{0}=\frac{1-\theta}{2(2-\theta)^{2}}
$$

In the subgame where the retailer accepts $M_{i}$ 's proposal, equilibrium profits are given by

$$
\begin{aligned}
\Pi_{R}^{i} & =\frac{1}{2(2-\theta)^{2}}+\frac{1+\rho_{i}}{4(2-\theta)^{2}} e_{i}+\frac{\left(4+5 \theta^{2}\right)\left(1+\rho_{i}^{2}\right)+2 \theta\left(8+\theta^{2}\right) \rho_{i}}{16(1+\theta)\left(4-\theta^{2}\right)^{2}} e_{i}^{2} \\
\Pi_{i}^{i} & =\frac{(1-\theta)\left(2(2+\theta)+\left(2+\theta \rho_{i}\right) e_{i}\right)^{2}}{8\left(4-\theta^{2}\right)^{2}}-\frac{1}{2}\left(1+\frac{k}{1-k}\left(1+\rho_{i}\right)^{2}\right) e_{i}^{2} \\
\Pi_{j}^{i} & =\frac{(1-\theta)\left(2(2+\theta)+\left(\theta+2 \rho_{i}\right) e_{i}\right)^{2}}{8\left(4-\theta^{2}\right)^{2}}
\end{aligned}
$$

Proof is essentially the same as for Lemma C.1.

Lemma C.3. For the setting in $\S 4$, in an equilibrium where $M_{i}$ 's proposal is accepted with positive probability, for $i, j \in\{1,2\}, i \neq j$, we require that:

(i) If $\Pi_{R}^{i *}=\Pi_{R}^{j *}$, then $\Pi_{i}^{i *}=\Pi_{i}^{j *}$ and $\Pi_{j}^{i *}=\Pi_{j}^{j *}$;

(ii) $\Pi_{R}^{i *} \geq \max \left\{\Pi_{R}^{0 *}, \Pi_{R}^{j *}\right\}$;

(iii) If $\Pi_{R}^{j *}<\Pi_{R}^{0 *}$, then $\Pi_{i}^{i *} \geq \Pi_{i}^{0 *}$, else $\Pi_{i}^{i *} \geq \Pi_{i}^{j *}$;

(iv) $\left(e_{i}^{*}, \rho_{i}^{*}\right) \in \underset{e_{i}, \rho_{i}}{\operatorname{argmax}} \Pi_{i}^{i}$,

$$
\text { s.t. } \quad \Pi_{R}^{i} \geq \max \left\{\Pi_{R}^{0 *}, \Pi_{R}^{j *}\right\}
$$

(v) $\Pi_{j}^{j *} \geq \max _{e_{j}, \rho_{j}} \Pi_{j}^{j}$,

$$
\text { s.t. } \Pi_{R}^{j} \geq \max \left\{\Pi_{R}^{0 *}, \Pi_{R}^{i *}\right\} .
$$

These conditions are also sufficient.

We first show that these conditions are necessary. We note that (C.8) is the same as (17). Consider an equilibrium where $\Pi_{R}^{i *}=\Pi_{R}^{j *}$. In such an equilibrium, the retailer chooses either 
manufacturer with equal probability. Thus, we have

$$
\Omega_{i}^{*}=\frac{1}{2}\left[\Pi_{i}^{i *}+\Pi_{i}^{j *}\right] \text { and } \Omega_{j}^{*}=\frac{1}{2}\left[\Pi_{j}^{i *}+\Pi_{j}^{j *}\right]
$$

Suppose $\Pi_{i}^{i *}>\Pi_{i}^{j *}$. Then, $M_{i}$ can strictly increase its profits by making a slightly better offer than $M_{j}$, such that the retailer accepts only $M_{i}$ 's proposal, which is a contradiction ${ }^{20}$. Similarly, if $\Pi_{i}^{i *}<\Pi_{i}^{j *}$, then $M_{i}$ can strictly increase its profits by making a slightly less attractive offer than $M_{j}$. Therefore, we have,

$$
\Pi_{R}^{i *}=\Pi_{R}^{j *} \Longrightarrow \Pi_{i}^{i *}=\Pi_{i}^{j *}
$$

Further, the same holds true from $M_{j}$ 's perspective as well. Thus, we have,

$$
\Pi_{R}^{i *}=\Pi_{R}^{j *} \Longrightarrow \Pi_{j}^{i *}=\Pi_{j}^{j *}
$$

Thus, (C.7) is a necessary condition.

Now consider an equilibrium where $M_{i}$ is chosen with positive probability. We have,

$$
\Omega_{i}^{*}=\Pi_{i}^{i^{*}} \text { and } \Omega_{j}^{*}=\Pi_{j}^{i^{*}}
$$

This follows immediately if $\Pi_{R}^{i *}>\Pi_{R}^{j *}$, since the retailer only chooses $M_{i}$. On the other hand, if $\Pi_{R}^{i *}=\Pi_{R}^{j *}$, then (C.15) follows from (C.7). Consequently, (C.9) is equivalent to (16a) and (16b).

From (C.15) and (18), we have that $\forall\left(e_{j}, \rho_{j}\right)$ s.t. $\Pi_{R}^{j}\left(e_{j}, \rho_{j}\right) \geq \max \left\{\Pi_{R}^{0 *}, \Pi_{R}^{i *}\right\}$,

$$
\begin{aligned}
& \Pi_{j}^{i^{*}} \geq \Omega_{j}\left(e_{i}^{*}, \rho_{i}^{*}, e_{j}, \rho_{j}\right), \\
\Longleftrightarrow & \Pi_{j}^{i^{*}} \geq \begin{cases}\Pi_{j}^{j}\left(e_{j}, \rho_{j}\right) & \text { if } \Pi_{R}^{j}\left(e_{j}, \rho_{j}\right)>\Pi_{R}^{i *} ; \\
\frac{1}{2}\left[\Pi_{j}^{i^{*}}+\Pi_{j}^{j}\left(e_{j}, \rho_{j}\right)\right] & \text { if } \Pi_{R}^{j}\left(e_{i}, \rho_{i}\right)=\Pi_{R}^{i *},\end{cases} \\
\Longleftrightarrow & \Pi_{j}^{i^{*}} \geq \Pi_{j}^{j}\left(e_{j}, \rho_{j}\right) .
\end{aligned}
$$

Therefore, (18) is equivalent to (C.11).

\footnotetext{
${ }^{20}$ For $\varepsilon>0$, sufficiently small, we have (a) $\Pi_{R}^{i}\left(e_{i}^{*}+\varepsilon, \rho_{i}^{*}\right)>\max \left\{\Pi_{R}^{0 *}, \Pi_{R}^{j *}\right\}$, since from (C.2) $\Pi_{R}^{i}$ is strictly increasing in $e_{i}$, and (b) $\Omega_{i}\left(e_{i}^{*}+\varepsilon, \rho_{i}^{*}, e_{j}^{*}, \rho_{j}^{*}\right)=\Pi_{i}^{i}\left(e_{i}^{*}+\varepsilon, \rho_{i}^{*}\right)>\frac{1}{2}\left[\Pi_{i}^{i *}+\Pi_{i}^{j *}\right]=\Omega_{i}^{*}$, since $\Pi_{i}^{i}$ is continuous in $e_{i}$ and $\Pi_{i}^{i *}>\Pi_{i}^{j *}$. Therefore, $M_{i}$ has an incentive to deviate.
} 
From (C.15) and (17), we have that $\forall\left(e_{i}, \rho_{i}\right)$ s.t. $\Pi_{R}^{i}\left(e_{i}, \rho_{i}\right) \geq \max \left\{\Pi_{R}^{0 *}, \Pi_{R}^{j *}\right\}$,

$$
\begin{aligned}
\Pi_{i}^{i^{*}} \geq \Omega_{i}\left(e_{i}, \rho_{i}, e_{j}^{*}, \rho_{j}^{*}\right), \\
\Longrightarrow \quad \Pi_{i}^{i^{*}} \geq\left\{\begin{array}{lll}
\Pi_{i}^{i}\left(e_{i}, \rho_{i}\right) & \text { if } & \Pi_{R}^{i}\left(e_{i}, \rho_{i}\right)>\Pi_{R}^{j *} ; \\
\frac{1}{2}\left[\Pi_{i}^{i}\left(e_{i}, \rho_{i}\right)+\Pi_{i}^{j^{*}}\right] & \text { if } & \Pi_{R}^{i}\left(e_{i}, \rho_{i}\right)=\Pi_{R}^{j *} .
\end{array}\right.
\end{aligned}
$$

Now, suppose there exists $\left(e_{i}^{\prime}, \rho_{i}^{\prime}\right)$ such that $\Pi_{R}^{i}\left(e_{i}^{\prime}, \rho_{i}^{\prime}\right) \geq \max \left\{\Pi_{R}^{0 *}, \Pi_{R}^{j *}\right\}$ and $\Pi_{R}^{i}\left(e_{i}^{\prime}, \rho_{i}^{\prime}\right)=\Pi_{R}^{j *}$ but $\Pi_{i}^{i^{*}}<\Pi_{i}^{i}\left(e_{i}^{\prime}, \rho_{i}^{\prime}\right)$. Then it must be possible for $M_{i}$ to increase its profits from $\Pi_{i}^{i *}$ by making a slightly better proposal than $\left(e_{i}^{\prime}, \rho_{i}^{\prime}\right)$, which is a contradiction ${ }^{21}$. Thus, we have that,

$$
\forall\left(e_{i}, \rho_{i}\right) \text { s.t. } \Pi_{R}^{i}\left(e_{i}, \rho_{i}\right) \geq \max \left\{\Pi_{R}^{0 *}, \Pi_{R}^{j *}\right\}, \Pi_{i}^{i *} \geq \Pi_{i}^{i}\left(e_{i}, \rho_{i}\right)
$$

In particular, (C.10) must hold since, by assumption, $\Pi_{R}^{i}\left(e_{i}^{*}, \rho_{i}^{*}\right) \geq \max \left\{\Pi_{R}^{0 *}, \Pi_{R}^{j *}\right\}$. Thus, we have shown that the conditions in Lemma C.3 are necessary.

To establish sufficieny, suppose that (C.7) to (C.11) hold for some pair of proposals $\left(\hat{e}_{i}, \hat{\rho}_{i}\right)$ and $\left(\hat{e}_{j}, \hat{\rho}_{j}\right)$ and $\Pi_{R}^{i}\left(\hat{e}_{i}, \hat{\rho}_{i}\right) \geq \Pi_{R}^{j}\left(\hat{e}_{j}, \hat{\rho}_{j}\right)$. We have,

$$
\Omega_{i}\left(\hat{e}_{i}, \hat{\rho}_{i}, \hat{e}_{j}, \hat{\rho}_{j}\right)=\Pi_{i}^{i}\left(\hat{e}_{i}, \hat{\rho}_{i}\right) \text { and } \Omega_{j}\left(\hat{e}_{i}, \hat{\rho}_{i}, \hat{e}_{j}, \hat{\rho}_{j}\right)=\Pi_{j}^{j}\left(\hat{e}_{j}, \hat{\rho}_{j}\right)
$$

This follows immediately if $\Pi_{R}^{i}\left(\hat{e}_{i}, \hat{\rho}_{i}\right)>\Pi_{R}^{j}\left(\hat{e}_{j}, \hat{\rho}_{j}\right)$, and follows from (C.7) if $\Pi_{R}^{i}\left(\hat{e}_{i}, \hat{\rho}_{i}\right)=$ $\Pi_{R}^{j}\left(\hat{e}_{j}, \hat{\rho}_{j}\right)$.

It follows that (C.9) is equivalent to (16a) and (16b). As in (C.16), it also follows that (C.11) is equivalent to (18). Now, consider any $\left(e_{i}, \rho_{i}\right)$ s.t. $\Pi_{R}^{i}\left(e_{i}, \rho_{i}\right) \geq \max \left\{\Pi_{R}^{0 *}, \Pi_{R}^{j}\left(\hat{e}_{j}, \hat{\rho}_{j}\right)\right\}$. From

\footnotetext{
${ }^{21}$ For $\varepsilon>0$, sufficiently small, we have (a) $\Pi_{R}^{i}\left(e_{i}^{\prime}+\varepsilon, \rho_{i}^{\prime}\right)>\max \left\{\Pi_{R}^{0 *}, \Pi_{R}^{j *}\right\}$, since from (C.2) $\Pi_{R}^{i}$ is strictly increasing in $e_{i}$, and (b) $\Omega_{i}\left(e_{i}^{\prime}+\varepsilon, \rho_{i}^{\prime}, e_{j}^{*}, \rho_{j}^{*}\right)=\Pi_{i}^{i}\left(e_{i}^{\prime}+\varepsilon, \rho_{i}^{\prime}\right)>\Pi_{i}^{i *}=\Omega_{i}^{*}$, since $\Pi_{i}^{i}$ is continuous in $e_{i}$ and $\Pi_{i}^{i}\left(e_{i}^{\prime}, \rho_{i}^{\prime}\right)>$ $\Pi_{i}^{i *}$. Therefore, $M_{i}$ has an incentive to deviate.
} 
(C.10) and (C.9) we have that,

$$
\begin{gathered}
\Pi_{i}^{i}\left(\hat{e}_{i}, \hat{\rho}_{i}\right) \geq \Pi_{i}^{j}\left(\hat{e}_{j}, \hat{\rho}_{j}\right) \text { and } \Pi_{i}^{i}\left(\hat{e}_{i}, \hat{\rho}_{i}\right) \geq \Pi_{i}^{i}\left(e_{i}, \rho_{i}\right) \\
\Longrightarrow \Pi_{i}^{i}\left(\hat{e}_{i}, \hat{\rho}_{i}\right) \geq\left\{\begin{array}{l}
\Pi_{i}^{i}\left(e_{i}, \rho_{i}\right) \\
\frac{1}{2}\left[\Pi_{i}^{i}\left(e_{i}, \rho_{i}\right)+\Pi_{i}^{j}\left(\hat{e}_{j}, \hat{\rho}_{j}\right)\right] \quad \text { if } \quad \Pi_{R}^{i}\left(e_{i}, \rho_{i}\right)>\Pi_{R}^{j}\left(\hat{e}_{j}, \hat{\rho}_{j}\right)=\Pi_{R}^{j}\left(\hat{e}_{j}, \hat{\rho}_{j}\right),
\end{array}\right. \\
\Longrightarrow \Pi_{i}^{i}\left(\hat{e}_{i}, \hat{\rho}_{i}\right) \geq \underset{e_{i}, \rho_{i}}{\max } \Omega_{i}, \\
\quad \text { s.t. } \Pi_{R}^{i} \geq \max \left\{\Pi_{R}^{0 *}, \Pi_{R}^{j}\left(\hat{e}_{j}, \hat{\rho}_{j}\right)\right\}, \\
\Longrightarrow \quad\left(\hat{e}_{i}, \hat{\rho}_{i}\right) \in \underset{e_{i}, \rho_{i}}{\operatorname{argmax}} \Omega_{i}, \\
\text { s.t. } \quad \Pi_{R}^{i} \geq \max \left\{\Pi_{R}^{0 *}, \Pi_{R}^{j}\left(\hat{e}_{j}, \hat{\rho}_{j}\right)\right\},
\end{gathered}
$$

since $\Pi_{R}^{i}\left(\hat{e}_{i}, \hat{\rho}_{i}\right) \geq \max \left\{\Pi_{R}^{0 *}, \Pi_{R}^{j}\left(\hat{e}_{j}, \hat{\rho}_{j}\right)\right\}$ by assumption. Thus, (17) also holds. Hence the conditions in Lemma C.3 are sufficient to establish the existence of an equilibrium.

Lemma C.4. For the setting in $\$ 5$, in the subgame starting at Stage 3 where the retailer has chosen to jointly engage both manufacturers $(r=J)$, the equilibrium service strategies are given by,

$$
e_{1}^{J *}=e_{2}^{J *}=\frac{2 k(1-\theta)(2-\theta) \mu}{4 k(2+\theta)(2-\theta)^{2}-\mu(1-k)(1-\theta) \theta}, \rho_{1}^{J *}=\rho_{2}^{J *}=\frac{\theta-2 k}{k(2-\theta)},
$$

and the equilibrium profits are given by,

$$
\begin{aligned}
\Pi_{1}^{J *}=\Pi_{2}^{J *} & =\frac{2 k(1-\theta)\left((1-\theta)\left(4 k(1-\theta)+\theta^{2}\right) \mu-4 k\left(4-\theta^{2}\right)^{2}\right)}{\left(4 k(2+\theta)(2-\theta)^{2}-\mu(1-k)(1-\theta) \theta\right)^{2}}, \\
\Pi_{R}^{J *} & =\frac{8 k^{2}\left(4-\theta^{2}\right)^{2}}{\left(k\left(32-15 \theta-9 \theta^{2}+4 \theta^{3}\right)-\theta(1-\theta)\right)^{2}} .
\end{aligned}
$$

Given the service $\left(e_{i}^{J}, \rho_{i}^{J}\right)$ by $M_{i}$ under joint assignment, and solving for the equilibrium retail 
and wholesale prices in the usual manner, we have,

$$
\begin{aligned}
& w_{1}=\frac{(1-\theta)\left(2(2+\theta)+\left(2+\theta \rho_{1}^{J}\right) e_{1}^{J}+\left(2 \rho_{2}^{J}+\theta\right) e_{2}^{J}\right)}{2\left(4-\theta^{2}\right)}, \\
& w_{2}=\frac{(1-\theta)\left(2(2+\theta)+\left(\theta+2 \rho_{1}^{J}\right) e_{1}^{J}+\left(2+\theta \rho_{2}^{J}\right) e_{2}^{J}\right)}{2\left(4-\theta^{2}\right)}, \\
& p_{1}=\frac{3-2 \theta}{2(2-\theta)}+\frac{3\left(2-\theta^{2}\right)+\left(5-2 \theta^{2}\right) \theta \rho_{1}^{J}}{4(1+\theta)\left(4-\theta^{2}\right)} e_{1}^{J}+\frac{\left(5-2 \theta^{2}\right) \theta+3\left(2-\theta^{2}\right) \rho_{2}^{J}}{4(1+\theta)\left(4-\theta^{2}\right)} e_{2}^{J}, \\
& p_{2}=\frac{3-2 \theta}{2(2-\theta)}+\frac{\left(5-2 \theta^{2}\right) \theta+3\left(2-\theta^{2}\right) \rho_{1}^{J}}{4(1+\theta)\left(4-\theta^{2}\right)} e_{1}^{J}+\frac{3\left(2-\theta^{2}\right)+\left(5-2 \theta^{2}\right) \theta \rho_{2}^{J}}{4(1+\theta)\left(4-\theta^{2}\right)} e_{2}^{J} .
\end{aligned}
$$

Solving for the equilibrium in service strategies in the usual manner, we obtain the result stated in the Lemma above.

\section{Technical Appendix}

\section{When Manufacturers Commit to Retail Category Profits}

We consider the setting with competition for category captaincy when joint service is not feasible. Let $\eta_{i}$ denote the retail category profits that $M_{i}$ commits to in its category captaincy proposal. We model the interactions in this setting as follows:

Stage 1 Proposal for category captaincy: $M_{1}, M_{2}$ simultaneously propose the minimum retail category profits $\eta_{i}$ if selected as the category captain.

Stage 2 Category captaincy decision: Retailer either accepts one of their proposals or rejects both. We denote this decision by $r \in\{0,1,2\}$, with $r=i$ if $M_{i}$ 's proposal is accepted, $i \in\{1,2\}$, and $r=0$ otherwise.

Stage 3 Service provision: If $M_{i}$ is chosen as the category captain, then it decides the service $\left(e_{i}, \rho_{i}\right)$ to provide, such that the retail category profits are at least $\eta_{i}$. If neither manufacturer was chosen as the category captain, then there is no service provision.

Stage 4 Wholesale price decisions: Manufacturers simultaneously set their wholesale price $w_{1}, w_{2}$. If $M_{i}$ was chosen as the category captain, then it ensures that the retail category profits are at least $\eta_{i}$.

Stage 5 Retail price decision: Retailer sets retail prices $p_{i}$. 
We note that a manufacturer decides the service in Stage 3 only if its proposal is accepted. This per se does not alter the analysis, since even earlier the retailer considered only its profits from the proposed service. However, we now also have that when wholesale prices are set in Stage 4, the category captain must keep in mind the retail profit commitment. This was not the case earlier, and this can result in a different outcome. This constraint also considerably complicates the analysis. Let $M_{i}$ 's equilibrium proposal be $\eta_{i}^{*}$. The rest of the notation is as defined in $\S 4$ in the main text.

We now derive the conditions for an equilibrium where $M_{i}$ 's proposal is accepted with positive probability. Consider Stage 4 in the subgame when $M_{i}$ 's proposal is accepted. $M_{i}$ and $M_{j}$, respectively, decide their wholesale prices as follows,

$$
\begin{array}{ll}
\max _{w_{i}} & \Pi_{i}^{i}, \\
\text { s.t. } & \Pi_{R}^{i} \geq \eta_{i}^{*}, \\
\max _{w_{j}} & \Pi_{j}^{i} .
\end{array}
$$

Therefore, while $M_{j}$ 's best response is as before, $M_{i}$ 's best response differs when the constraint is binding. Thus, given $\left(e_{i}, \rho_{i}\right)$, the equilibrium outcome of the subgame starting at Stage 4 can be different than in the earlier situation. In Stage $3, M_{i}$ 's service decision is given by

$$
\max _{e_{i}, \rho_{i}}\left\{\begin{array}{ll}
\max _{w_{i}} & \Pi_{i}^{i}, \\
\text { s.t. } & \Pi_{R}^{i} \geq \eta_{i}^{*}
\end{array}\right\} .
$$

We note that the two maximization problems cannot be collapsed in to one even though $M_{i}$ sets all decision variables, since $M_{j}$ sets its wholesale price simultaneously with $M_{i}$ 's but after observing the retail service environment.

For a given level of retail profits $\eta_{i}$, the above programming problem will yield a different optimal service $\left(e_{i}, \rho_{i}\right)$ than in our earlier setting unless if for this optimum service, the retail profit constraint is only weakly binding when setting wholesale price. Thus, in general, the optimum service differs from that in our earlier analysis and, therefore, may result in a different equilibrium outcome. However, as we will see, for some $(\theta, k)$ we continue to have the same equilibrium outcome. 
In Stage 2, the retailer chooses the proposal that provides it the most profits, or chooses either proposal with equal probability if both offer the same profits. Thus, we have

$$
\eta_{i}^{*} \geq \max \left\{\Pi_{R}^{0 *}, \eta_{j}^{*}\right\}
$$

In Stage 1 , for $\eta_{i}^{*}$ to be $M_{i}$ 's best response, we require,

$$
\begin{aligned}
& \Pi_{i}^{i}\left(\eta_{\iota}^{*}\right) \geq \Pi_{i}^{j}\left(\eta_{j}^{*}\right), \\
& \eta_{i}^{*} \in \underset{\eta_{i}^{*} \geq \max \left\{\Pi_{R}^{0 *}, \eta_{j}^{*}\right\}}{\operatorname{argmax}}\left\{\max _{e_{i}, \rho_{i}}\left\{\begin{array}{cl}
\max _{w_{i}} & \Pi_{i}^{i}, \\
\text { s.t. } & \Pi_{R}^{i} \geq \eta_{i}^{*}
\end{array}\right\}\right\} .
\end{aligned}
$$

We also require that $M_{j}$ does not have an incentive to outbid $M_{i}$,

$$
\Pi_{j}^{i}\left(\eta_{i}^{*}\right) \geq \max _{\eta_{j} \geq \max \left\{\Pi_{R}^{0 *}, \eta_{i}^{*}\right\}}\left\{\max _{e_{j}, \rho_{j}}\left\{\begin{array}{cl}
\max _{w_{j}} & \Pi_{j}^{j}, \\
\text { s.t. } & \Pi_{R}^{j} \geq \eta_{j}
\end{array}\right\}\right\}
$$

As before, we can define the notion of active competition. It follows from essentially the same arguments as before that in the absence of active competition, since $M_{i}$ 's proposal is not driven by $M_{j}$ 's proposal and since the retailer will accept any proposal that induces some service, $M_{i}$ 's proposal maximizes its own profits, i.e., it is the solution of unconstrained maximization of $M_{i}$ 's profits, which is the same as in the earlier setting. Therefore, any equilibrium without active competition from our earlier analysis is also an equilibrium of this game.

In the case of equilibrium with active competition, we can again show that manufacturers bid up the retail profits up to the point where they are indifferent between winning and losing category captaincy, and both manufacturers realize the same profits. So we have,

$$
\begin{aligned}
\eta_{1}^{*} & =\eta_{2}^{*}, \\
\Pi_{1}^{1}\left(\eta_{1}^{*}\right) & =\Pi_{1}^{2}\left(\eta_{2}^{*}\right), \Pi_{2}^{2}\left(\eta_{2}^{*}\right)=\Pi_{2}^{1}\left(\eta_{1}^{*}\right), \\
\Pi_{1}^{1}\left(\eta_{1}^{*}\right) & =\Pi_{2}^{2}\left(\eta_{2}^{*}\right) .
\end{aligned}
$$


Now, any equilibrium with active competition from our earlier analysis (in §4) would continue to be an equilibrium (with active competition) of this game if and only if for the equilibrium level of retail profits, the optimal service in the current setting (as given by (T.3)) is the same as in the earlier setting. In other words, for this level of retail profits, the additional degree of freedom of being able to set also the wholesale price to ensure a certain level of retail profits does not matter. We can verify this numerically.

On the other hand, suppose in an equilibrium with active competition the constraint does matter. Consider the programming problem in (T.3). Since $w_{i}$ is an (implict) function of $\left(e_{i}, \rho_{i}\right)$, we have

$$
\begin{aligned}
\frac{\partial \Pi_{i}^{i}}{\partial e_{i}}+\frac{\partial \Pi_{i}^{i}}{\partial w_{i}} \frac{\partial w_{i}}{\partial e_{i}} & =0 \\
\frac{\partial \Pi_{i}^{i}}{\partial \rho_{i}}+\frac{\partial \Pi_{i}^{i}}{\partial w_{i}} \frac{\partial w_{i}}{\partial \rho_{i}}-\mu & =0 \\
\mu \geq 0, \text { and } \mu=0 \text { if } \rho_{i} & <1 .
\end{aligned}
$$

Since the retail profit constraint is binding, we also have

$$
\begin{aligned}
& \frac{\partial \Pi_{R}^{i}}{\partial w_{i}} \frac{\partial w_{i}}{\partial e_{i}}+\frac{\partial \Pi_{R}^{i}}{\partial e_{i}}=0, \\
& \frac{\partial \Pi_{R}^{i}}{\partial w_{i}} \frac{\partial w_{i}}{\partial e_{i}}+\frac{\partial \Pi_{R}^{i}}{\partial e_{i}}=0 .
\end{aligned}
$$

Finally, from (T.2) we have

$$
w_{j}=\frac{(1-\theta)\left(1+\frac{1}{2} e_{i} \rho_{i}\right)}{2}+\frac{\theta}{2} w_{i}
$$

We can numerically solve for such an equilibrium from (T.11) to (T.16).

We find that in some instances there are multiple equilibria. To the extent that the manufacturers are able to co-ordinate on the equilibrium when making their proposals in Stage 1, we select the equilibrium that is Pareto-dominant for the manufacturers. We find that whenever an equilibrium from the earlier setting (as in $§ 4$ ) is also an equilibrium of this setting, then this equilibrium is Pareto-dominant.

Figure T.1 depicts the equilibrium outcome for this setting. Whenever the equilibrium outcome is differen than that in $\S 4$, we find that the competition for category captaincy is more intense and 
the service provided is less-biased than in the setting in $\S 4$.

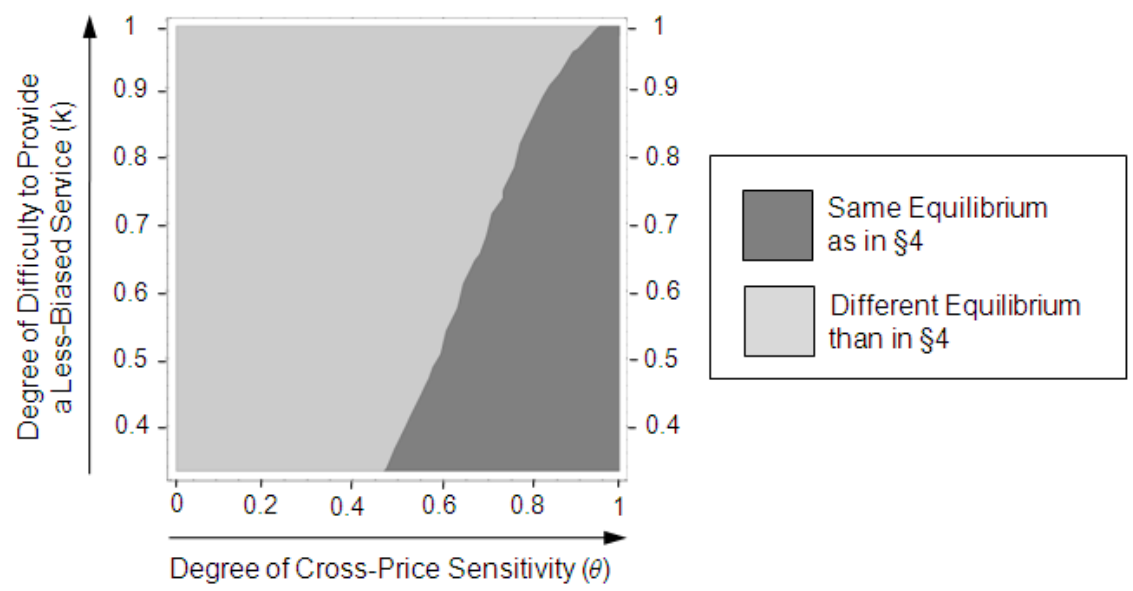

Figure T.1: Equilibrium Outcome when Manufacturers Commit to Retail Category Profits.

\section{Competition for Category Captaincy Between Asymmetric Manufacturers}

When manufacturers are asymmetric on some dimension, as in $\S 6.1$ or $\S 6.2$, the equilibrium conditions in (15) to (18) still apply, except that when the retailer is indifferent between the two proposals, the choice of category captain is now also decided in equilibrium. In the analysis here, we only consider equilibria where the retailer uses pure strategies when selecting the category captain. When manufacturers are asymmetric, it is straightforward to verify that almost always only equilibria in pure strategies exist ${ }^{22,23}$. Therefore, for an equilibrium where $M_{i}$ is chosen as the category captain, the necessary and sufficient conditions are

$$
\begin{aligned}
& \Pi_{R}^{i *} \geq \max \left\{\Pi_{R}^{0 *}, \Pi_{R}^{j *}\right\}, \\
& \text { if } \Pi_{R}^{j *}<\Pi_{R}^{0 *} \text {, then } \Pi_{i}^{i *} \geq \Pi_{i}^{0 *} \text {, else } \Pi_{i}^{i *} \geq \Pi_{i}^{j *}, \\
& \left(e_{i}^{*}, \rho_{i}^{*}\right) \in \underset{e_{i}, \rho_{i}}{\operatorname{argmax}} \Pi_{i}^{i}, \\
& \text { s.t. } \Pi_{R}^{i} \geq \max \left\{\Pi_{R}^{0 *}, \Pi_{R}^{j *}\right\}, \\
& \Pi_{j}^{i *} \geq \max _{e_{j}, \rho_{j}} \quad \Pi_{j}^{j}, \\
& \text { s.t. } \quad \Pi_{R}^{j} \geq \max \left\{\Pi_{R}^{0 *}, \Pi_{R}^{i *}\right\} .
\end{aligned}
$$

\footnotetext{
${ }^{22}$ There might only be a measure zero set of $(\theta, k)$ for which an equilibrium exists where the retailer mixes when indifferent between proposals.

${ }^{23}$ Whenever an equilibrium where the retailer uses a mixed strategy exists, there is a corresponding pair of equilibria in pure strategy that leads to the same equilibrium outcome in terms of profits and differ in the choice of category captain.
} 
We consider only equilibria with active competition. Since, from the definition of active competition, $M_{i}$ 's proposal must be driven by the prospect of losing category captaincy to its rival, we have

$$
\Pi_{R}^{i *}=\Pi_{R}^{j *}>\Pi_{R}^{0 *}
$$

We restrict attention to equilibria where weakly dominated strategies are not used. In particular, this requires that $M_{j}$ 's strategy is robust to small trembles in $M_{i}$ 's proposal. This leads to stronger equilibrium conditions that ensure uniqueness. First, we require that though $M_{j}$ 's proposal is not accepted in equilibrium, still $M_{j}$ 's proposal maximizes its profits conditional on the retailer accepting it:

$$
\begin{aligned}
\left(e_{j}^{*}, \rho_{j}^{*}\right) \in \underset{e_{j}, \rho_{j}}{\operatorname{argmax}} & \Pi_{j}^{j}, \\
& \text { s.t. } \quad \Pi_{R}^{j}=\Pi_{R}^{j *} .
\end{aligned}
$$

Second, we also require that $M_{j}$ is strictly indifferent between winning and losing category captaincy:

$$
\Pi_{j}^{i *}=\Pi_{j}^{j *}
$$

From these equilibrium conditions, we solve for the equilibrium numerically for the settings where the manufacturers are asymmetric in demand (§6.1) or asymmetric in cost $(\S 6.2)$. In the latter case, we find that there is always a unique equilibrium with active competition and the more efficient manufacturer is the category captain. In the former case, we find that there is a unique equilibrium almost always (i.e., the equilibrium is unique except on the boundary between the regions where $M_{1}$ and $M_{2}$ are chosen as the catergoy captains), and that the smaller manufacturer may, in fact, become the category captain. This is depicted in Figure 7 in the main text. 